\title{
1 Supplemental Information
}

\section{Methods}

3 Chemical analysis: Quantitative analysis for PCB congeners and selected chlorinated pesticides

4 was performed on an Agilent 6890 Gas Chromatograph equipped with an electron capture

5 detector. A J\&W DB-5 60m fused silica capillary column (0.25 $\mu \mathrm{m}$ film thickness, $0.25 \mathrm{~mm}$ i.d.)

6 was used to achieve the separation. Ultra-high purity helium was the carrier gas (flow rate $=1.3$

$7 \mathrm{ml} / \mathrm{min}$ ) and 95\%:5\% Argon/Methane (P5) was used as the auxiliary gas. The initial column

8 temperature was held at $90^{\circ} \mathrm{C}$ for $1 \mathrm{~min}$, increased to $170^{\circ} \mathrm{C}$ at $25^{\circ} \mathrm{C} \mathrm{min}{ }^{-1}$ and then to $215^{\circ} \mathrm{C}$ at

$9 \quad 1.5^{\circ} \mathrm{C} \min ^{-1}$. After $3 \mathrm{~min}$ at $215^{\circ} \mathrm{C}$, the temperature was increased to $250^{\circ} \mathrm{C}$ at $1.5^{\circ} \mathrm{C} \mathrm{min}^{-1}$.

10 Final column temperature was taken to $300^{\circ} \mathrm{C}$ at $20^{\circ} \mathrm{C} \mathrm{min}{ }^{-1}$ and held for 5 minutes. The

11 injection port temperature was $250^{\circ} \mathrm{C}$ and the detector temperature was $300^{\circ} \mathrm{C}$. Integration and

12 calculations were accomplished by Agilent ChemStation ${ }^{\circledR}$ software.

14 For all PCB congener analyses, procedural blanks, standard reference materials [1], analytical

15 duplicates and matrix spiked samples were routinely taken through the entire analytical

16 procedure with each batch of 20 samples to evaluate and maintain quality assurance and quality

17 control. Procedural blanks were free of any contaminants; the laboratory values for standard

18 reference materials were within $30 \%$ of true values on average for most of the analytes. The

19 relative standard deviation for analytical duplicates was less than $10 \%$ for most analytes and the

20 range for matrix spike recoveries was 73\% to 113\%. The method detection limits (MDLs) for 18

21 PCB congeners in $4 \mathrm{~g}$ wet egg contents ranged from 0.06 to $0.11 \mathrm{ng} / \mathrm{g}$. 
22 Eight extracts were not analyzed for DLCs until 2014. To test for possible changes between

232005 and 2014, these extracts were reanalyzed for other PCB congeners. For 7 extracts, the

24 mean difference in Total PCBs between the measurements in 2005 and 2014 was $+3.6 \%$ with a

25 maximum difference of $+16 \%$. For the eighth extract, the difference between the two

26 measurements was $-46 \%$; data for this sample were not used.

28 To convert from dw to aww, we use the mean value of 0.24 for tern eggs derived from data in

29 [2]. Concentrations should not have been changed significantly by embryonic development in

30 the 1972 eggs that were hatched, because mass intake of $\mathrm{O}_{2}$ during embryonic development in

31 birds is approximately balanced by loss of $\mathrm{CO}_{2}$, so that the net loss of mass consists primarily of

$32 \mathrm{H}_{2} \mathrm{O}[3]$.

34 Tern breeding pair censuses: Data on the numbers of breeding pairs of common and roseate

35 terns at the nesting sites in Buzzards Bay, MA, were compiled from various sources [4 - 11].

36 Field methods were described in the sources cited as well as [12 -14]. 
1. Wise, S. A.; Poster, D. L.; Schantz, M. M.; Kucklick, J. R.; Sander, L. C.; Lopez de Alda, M.

41 J.; Schubert, P.; Parris, R. M.; Porter, B. J. Three new mussel tissue standard reference

42 materials (SRMs) for the determination of organic contaminants. Anal. Bioanal. Chem. 2004,

$43 \quad 378,1213-1231$.

44 2. Nisbet, I. C. T. Dependence of fledging success on egg-size, parental performance and egg-

45 composition among common and roseate terns, Sterna hirundo and S. dougallii. Ibis 1978, $46 \quad 110,205-214$.

47 3. Ar, A.; Rahn, H. Water in the avian egg: overall budget of incubation. Amer. Zool. 1980, $48 \quad 20,477-484$.

49 4. Nisbet, I. C. T.; Reynolds, L. M. Organochlorine residues in common terns and associated 50 estuarine organisms, Massachusetts, USA, 1971-81. Mar. Environ. Res. 1984, 11, 33-66.

51 5. Nisbet, I. C. T. Terns in Massachusetts: present numbers and historical changes. Bird$52 \quad$ Banding 1973, 44, 27-55.

53 6. Breton, A. R.; Nisbet, I. C. T.; Mostello, C. S.; Hatch, J. J. Age-dependent survival and 54 breeding dispersal within a metapopulation of common terns Sterna hirundo. Ibis, 2014, 156, $55 \quad 534-547$.

56 7. Nisbet, I. C. T.; Drury, W. H. Measuring breeding success in common and roseate terns. Bird$57 \quad$ Banding 1972, 43, 97-106.

58 8. Nisbet, I.C.T. Population models for common terns in Massachusetts. Bird-Banding 1978, $59 \quad 49,50-58$. 
60

61

62

63

64

65

66

67

68

69

70

71

72

73

74

75

76

77

78

79

80

81

82

9. Nisbet, I. C. T. Status and trends of the Roseate Tern Sterna dougallii in North America and the Caribbean. Report to U.S. Fish \& Wildlife Service, Office of Endangered Species. Newton Corner, Mass. 1980.

10. Nisbet, I. C. T. Biological characteristics of the Roseate Tern Sterna dougallii. Report to U.S. Fish \& Wildlife Service, Office of Endangered Species, Newton Corner, Mass. 1981.

11. Burger, J.; Nisbet, I. C. T.; Gochfeld, M. Temporal patterns in reproductive success of the endangered Roseate Tern (Sterna dougallii) nesting at Bird Island, Massachusetts, and Cedar Beach, New York. Auk 1996, 113, 131-142.

12. Austin, O. L. Site tenacity, a behaviour trait of the common tern (Sterna hirundo Linn.). Bird-banding 1949, 20, 1-39.

13. Austin, O. L. 1951. Group adherence in the Common Tern. Bird-Banding 1951, 22, 1-15.

14. Austin, O. L. The status of the Cape Cod terns in 1944: A behaviour study. Bird-Banding 1945, 16, 10-27.

15. Latimer, J. S.; Boothman, W. S.; Pesch, C. E.; Chmura, G. L.; Pospelova, V.; Jayaraman, S. Environmental stress and recovery: the geochemical record of human disturbance in New Bedford Harbor and Apponagansett Bay, Massachusetts (USA). Sci. Total Environ. 2003, 313, 153-176.

16. Hansen, B. G.; Paya-Perez, A. B.; Rahman, M.; Larsen, B. R. QSARs for Kow and Koc of PCB congeners: a critical examination of data, assumptions, and statistical approaches. Chemosphere 1999, 29, 2209-2228.

17. Howard, P.; Meylan, W. (Eds). Handbook of physical properties of organic chemicals. Boca Raton, FL: CRC Press, Lewis Publishers, 1997. 


\section{Supplemental Tables}

84 Table S1. Concentrations of PCB congeners and $p, p$ '-DDE in samples of tern eggs from 85 Buzzards Bay, Massachusetts, USA. All concentrations are reported in ng g ${ }^{-1}$ adjusted wet weight as described in Methods; when below minimum detection limit (MDL), values are shown in bold as $1 \frac{1}{2}$ MDL.

\begin{tabular}{|c|c|c|c|c|c|c|c|c|c|}
\hline Lab ID & Sample & Site & Year & Period & Species & PCB008 & PCB018 & PCB028 & PCB052 \\
\hline $30848 \mathrm{~A}$ & Egg & RI & 1972 & 1970s & $\mathrm{CT}$ & 195.60 & 123.06 & 2589.84 & 2154.12 \\
\hline 30953A & Egg & RI & 1972 & 1970s & CT & 34.43 & 54.62 & 2395.70 & 426.61 \\
\hline $30954 \mathrm{~A}$ & Egg & RI & 1972 & $1970 \mathrm{~s}$ & CT & 0.20 & 0.26 & 25.29 & 1.95 \\
\hline $30955 \mathrm{~A}$ & Egg & RI & 1972 & 1970s & $\mathrm{CT}$ & 0.10 & 0.13 & 74.38 & 5.67 \\
\hline 30957A & Egg & $\mathrm{RI}$ & 1972 & 1970s & CT & 63.85 & 6.69 & 3756.28 & 822.91 \\
\hline 30959A & Egg & RI & 1972 & $1970 \mathrm{~s}$ & CT & 233.53 & 61.12 & 4771.18 & 1646.84 \\
\hline 30960A & Egg & RI & 1972 & $1970 \mathrm{~s}$ & $\mathrm{CT}$ & 253.43 & 112.09 & 2659.47 & 1773.44 \\
\hline 30961A & Egg & RI & 1972 & 1970s & $\mathrm{CT}$ & 378.41 & 126.36 & 3293.37 & 1677.30 \\
\hline 30962A & Egg & RI & 1972 & 1970s & CT & 0.15 & 0.19 & 1045.43 & 612.81 \\
\hline 30963A & Egg & $\mathrm{RI}$ & 1972 & 1970s & CT & 691.43 & 225.20 & 4662.70 & 2266.46 \\
\hline 30970A & Egg & RI & 1972 & 1970s & $\mathrm{CT}$ & 52.21 & 7.99 & 1447.22 & 966.42 \\
\hline 30956A & Egg & $\mathrm{RI}$ & 1972 & 1970s & RT & 0.18 & 0.17 & 509.37 & 60.85 \\
\hline $30712 \mathrm{~A}$ & Egg & $\mathrm{BI}$ & 1994 & 1990s & $\mathrm{RT}$ & 3.26 & 0.06 & 16.17 & 3.05 \\
\hline 30713A & Egg & $\mathrm{BI}$ & 1994 & 1990s & $\mathrm{RT}$ & 2.17 & 0.08 & 5.98 & 0.85 \\
\hline $30736 \mathrm{~A}$ & Egg & $\mathrm{BI}$ & 1994 & 1990s & $\mathrm{RT}$ & 6.35 & 0.07 & 12.79 & 2.68 \\
\hline $30743 \mathrm{~A}$ & Egg & $\mathrm{BI}$ & 1994 & 1990s & $\mathrm{RT}$ & 0.78 & 0.07 & 84.07 & 14.19 \\
\hline $30749 \mathrm{~A}$ & Egg & $\mathrm{BI}$ & 1994 & 1990s & RT & 3.48 & 0.07 & 22.57 & 3.07 \\
\hline $30755 \mathrm{~A}$ & Egg & $\mathrm{BI}$ & 1994 & 1990s & $\mathrm{RT}$ & 0.44 & 0.08 & 23.25 & 2.38 \\
\hline 30761A & Egg & $\mathrm{BI}$ & 1994 & 1990s & RT & 9.80 & 0.07 & 25.15 & 20.33 \\
\hline $30767 \mathrm{~A}$ & Egg & $\mathrm{BI}$ & 1994 & 1990s & $\mathrm{RT}$ & 6.84 & 0.08 & 9.70 & 4.24 \\
\hline $30721 \mathrm{~A}$ & Eggpool & $\mathrm{BI}$ & 1994 & 1990s & $\mathrm{RT}$ & 7.36 & 0.07 & 19.92 & 3.13 \\
\hline
\end{tabular}


90

91

\begin{tabular}{|r|r|r|r|r|r|r|r|r|}
\hline Lab ID & PCB044 & PCB066 & PCB101 & PCB118 & PCB153 & PCB105 & PCB138 & PCB187 \\
\hline 30848A & 368.12 & 2587.32 & 2596.16 & 4042.27 & 4332.28 & 1956.45 & 3907.43 & 753.32 \\
\hline 30953A & 94.68 & 2248.28 & 1329.78 & 3245.47 & 3133.78 & 1617.10 & 2879.43 & 408.70 \\
\hline 30954A & 5.06 & 57.36 & 13.46 & 252.91 & 762.52 & 80.47 & 491.53 & 222.38 \\
\hline 30955A & 0.78 & 124.33 & 85.84 & 218.18 & 342.78 & 83.79 & 268.34 & 64.56 \\
\hline 30957A & 19.25 & 3254.90 & 2403.33 & 4377.69 & 4154.69 & 2232.94 & 3629.41 & 565.81 \\
\hline 30959A & 71.26 & 3600.97 & 2877.73 & 3831.06 & 3685.06 & 1659.49 & 3163.75 & 495.50 \\
\hline 30960A & 620.88 & 2118.47 & 1919.25 & 2601.72 & 2684.19 & 1056.32 & 2296.07 & 326.35 \\
\hline 30961A & 288.44 & 2671.58 & 2170.07 & 3611.90 & 3614.34 & 1640.10 & 3179.44 & 451.81 \\
\hline 30962A & 118.95 & 1178.83 & 777.63 & 2785.27 & 3120.78 & 1179.87 & 2672.36 & 435.32 \\
\hline 30963A & 306.10 & 3954.36 & 3287.10 & 5020.99 & 4893.10 & 2450.49 & 4212.97 & 647.07 \\
\hline 30970A & 202.61 & 1138.65 & 939.49 & 1634.63 & 1604.06 & 827.23 & 1463.14 & 218.57 \\
\hline 30956A & 0.66 & 627.30 & 479.60 & 1303.93 & 1635.01 & 590.99 & 1375.52 & 383.98 \\
\hline 30712A & $\mathbf{0 . 0 3}$ & 39.96 & 85.91 & 208.19 & 402.17 & 44.12 & 266.78 & 63.45 \\
\hline 30713A & $\mathbf{0 . 0 3}$ & 14.92 & 17.57 & 116.39 & 291.55 & 21.77 & 178.99 & 48.03 \\
\hline 30736A & $\mathbf{0 . 0 3}$ & 24.23 & 41.80 & 179.89 & 392.21 & 34.14 & 251.38 & 66.76 \\
\hline 30743A & 0.74 & 193.18 & 200.92 & 719.99 & 1003.38 & 196.15 & 729.09 & 173.39 \\
\hline 30749A & $\mathbf{0 . 0 3}$ & 49.15 & 83.20 & 271.48 & 528.59 & 58.29 & 353.24 & 93.74 \\
\hline 30755A & $\mathbf{0 . 0 3}$ & 51.08 & 53.37 & 211.32 & 378.29 & 46.82 & 256.30 & 54.99 \\
\hline 30761A & 1.00 & 56.18 & 108.89 & 393.67 & 748.02 & 86.44 & 503.73 & 146.62 \\
\hline 30767A & $\mathbf{0 . 0 3}$ & 21.42 & 39.88 & 158.82 & 352.48 & 29.19 & 222.93 & 61.83 \\
\hline 30721A & $\mathbf{0 . 0 2}$ & 41.91 & 67.97 & 193.28 & 321.40 & 47.52 & 228.43 & 61.58 \\
\hline
\end{tabular}

Table S1 (continued).
92

93 


\section{Table S1 (continued).}

95

\begin{tabular}{|c|c|c|c|c|c|c|c|c|}
\hline Lab ID & PCB128 & PCB180 & PCB170 & PCB195 & PCB206 & PCB209 & Total PCBs & p,p'-DDE \\
\hline 30848A & 1269.33 & 1535.83 & 863.67 & 64.43 & 76.36 & 8.60 & 29424.18 & 985.86 \\
\hline 30953A & 966.53 & 1007.22 & 570.44 & 42.97 & 77.59 & 26.85 & 20560.17 & 677.29 \\
\hline 30954A & 71.62 & 421.86 & 139.84 & 29.16 & 27.88 & 24.29 & 2628.04 & 2515.14 \\
\hline 30955A & 42.01 & 167.77 & 49.16 & 7.00 & 13.80 & 4.63 & 1553.25 & 361.89 \\
\hline 30957A & 1244.02 & 1358.87 & 768.32 & 54.45 & 77.36 & 24.12 & 28814.89 & 864.28 \\
\hline 30959A & 831.81 & 1108.35 & 508.36 & 46.56 & 108.76 & 43.19 & 28744.49 & 779.46 \\
\hline 30960A & 519.75 & 694.40 & 315.84 & 17.96 & 30.34 & 2.39 & 20002.36 & 630.26 \\
\hline $30961 \mathrm{~A}$ & 941.42 & 1117.07 & 579.73 & 37.33 & 48.74 & 9.30 & 25836.72 & 856.63 \\
\hline 30962A & 822.41 & 1085.06 & 588.03 & 39.80 & 46.06 & 5.75 & 16514.71 & 565.93 \\
\hline 30963A & 1412.72 & 1762.98 & 950.32 & 99.66 & 175.90 & 62.35 & 37081.91 & 1186.65 \\
\hline 30970A & 504.45 & 576.36 & 347.02 & 28.05 & 28.56 & 3.91 & 11990.57 & 362.91 \\
\hline 30956A & 396.10 & 569.22 & 253.24 & 25.25 & 28.51 & 5.74 & 8245.62 & 597.09 \\
\hline 30712A & 50.87 & 75.56 & 28.84 & 2.45 & 5.77 & 1.96 & 1298.61 & 95.20 \\
\hline $30713 \mathrm{~A}$ & 29.04 & 62.40 & 23.09 & 1.97 & 6.09 & 2.06 & 822.99 & 36.95 \\
\hline 30736A & 46.55 & 79.00 & 32.08 & 2.50 & 5.90 & 1.77 & 1180.12 & 81.76 \\
\hline $30743 \mathrm{~A}$ & 187.54 & 211.03 & 96.40 & 6.33 & 8.42 & 1.84 & 3827.53 & 139.26 \\
\hline 30749A & 72.72 & 115.63 & 50.03 & 3.94 & 7.17 & 2.18 & 1718.59 & 117.32 \\
\hline 30755A & 51.28 & 63.36 & 28.08 & 1.77 & 3.66 & 0.65 & 1227.13 & 60.92 \\
\hline $30761 \mathrm{~A}$ & 108.24 & 172.11 & 75.17 & 5.17 & 8.07 & 2.22 & 2470.86 & 127.65 \\
\hline 30767A & 40.90 & 70.13 & 28.87 & 1.92 & 3.89 & 0.59 & 1053.73 & 56.20 \\
\hline $30721 \mathrm{~A}$ & 51.46 & 68.24 & 32.10 & 2.19 & 4.12 & 0.81 & 1151.51 & 90.18 \\
\hline
\end{tabular}

96 
98

99

Table S1 (continued).

\begin{tabular}{|l|r|r|r|r|r|r|r|r|r|}
\hline Lab ID & Sample & Site & Year & Period & Species & PCB008 & PCB018 & PCB028 & PCB052 \\
\hline 30714A & Eggpool & BI & 1995 & 1990s & CT & $\mathbf{0 . 0 6}$ & 1.22 & 61.84 & 26.91 \\
\hline 30722A & Eggpool & BI & 1995 & 1990s & CT & 7.77 & 18.58 & 299.21 & 264.39 \\
\hline 30724A & Eggpool & RI & 1995 & 1990s & CT & 4.69 & $\mathbf{0 . 0 6}$ & 28.00 & 2.84 \\
\hline 30737A & Eggpool & RI & 1995 & 1990s & CT & 5.79 & $\mathbf{0 . 0 8}$ & 15.49 & 5.27 \\
\hline 30715A & Eggpool & BI & 1996 & 1990s & CT & 1.98 & $\mathbf{0 . 0 7}$ & 28.84 & 6.49 \\
\hline 30744A & Eggpool & BI & 1996 & 1990s & CT & 5.94 & $\mathbf{0 . 0 6}$ & 17.06 & 17.95 \\
\hline 30725A & Egg & BI & 1996 & 1990s & RT & 4.13 & $\mathbf{0 . 0 7}$ & 3.94 & 0.12 \\
\hline 30750A & Egg & BI & 1996 & 1990s & RT & 5.47 & $\mathbf{0 . 0 8}$ & 7.30 & 5.77 \\
\hline 30762A & Egg & BI & 1996 & 1990s & RT & 1.46 & $\mathbf{0 . 0 8}$ & 20.52 & 3.51 \\
\hline 30773A & Egg & BI & 1996 & 1990s & RT & 4.37 & $\mathbf{0 . 0 9}$ & 2.56 & 0.04 \\
\hline 30781A & Egg & BI & 1996 & 1990s & RT & 2.56 & $\mathbf{0 . 0 8}$ & 21.24 & 1.69 \\
\hline 30788A & Egg & BI & 1996 & 1990s & RT & 1.31 & $\mathbf{0 . 0 9}$ & 7.57 & 1.29 \\
\hline 30796A & Egg & BI & 1996 & 1990s & RT & 6.72 & $\mathbf{0 . 0 8}$ & 18.90 & 3.73 \\
\hline 30803A & Egg & BI & 1996 & 1990s & RT & 4.32 & $\mathbf{0 . 0 8}$ & 13.96 & 6.13 \\
\hline 30731A & Eggpool & RI & 1996 & 1990s & CT & 3.55 & $\mathbf{0 . 0 7}$ & 35.01 & 25.61 \\
\hline 30738A & Egg & RI & 1996 & 1990s & RT & 7.54 & $\mathbf{0 . 0 7}$ & 17.09 & 8.07 \\
\hline 30756A & Egg & RI & 1996 & 1990s & RT & 2.11 & $\mathbf{0 . 0 8}$ & 8.11 & 0.98 \\
\hline 30768A & Egg & RI & 1996 & 1990s & RT & 4.10 & $\mathbf{0 . 0 7}$ & 90.35 & 56.22 \\
\hline 30778A & Egg & RI & 1996 & 1990s & RT & 11.71 & $\mathbf{0 . 0 8}$ & 25.61 & 4.15 \\
\hline 30785A & Egg & RI & 1996 & 1990s & RT & 5.90 & $\mathbf{0 . 0 8}$ & 10.35 & 2.09 \\
\hline 30792A & Egg & RI & 1996 & 1990s & RT & 5.53 & $\mathbf{0 . 0 8}$ & 7.21 & 0.73 \\
\hline 30799A & Egg & RI & 1996 & 1990s & RT & 4.74 & $\mathbf{0 . 0 7}$ & 16.18 & 0.03 \\
\hline Egg & RI & 1996 & 1990s & RT & 2.30 & $\mathbf{0 . 0 8}$ & 12.69 & 0.75 \\
\hline
\end{tabular}

100

101 
Table S1 (continued).

103

\begin{tabular}{|c|c|c|c|c|c|c|c|c|}
\hline Lab ID & PCB044 & PCB066 & PCB101 & PCB118 & PCB153 & PCB105 & PCB138 & PCB187 \\
\hline $30714 \mathrm{~A}$ & 2.92 & 66.07 & 123.57 & 255.49 & 431.76 & 54.78 & 292.94 & 59.64 \\
\hline $30722 \mathrm{~A}$ & 29.95 & 255.79 & 430.91 & 604.07 & 764.19 & 163.39 & 514.58 & 107.34 \\
\hline $30724 \mathrm{~A}$ & 0.02 & 41.18 & 55.72 & 288.55 & 534.19 & 61.22 & 345.69 & 71.58 \\
\hline 30737A & 0.40 & 36.28 & 54.00 & 196.60 & 360.07 & 44.13 & 237.17 & 63.00 \\
\hline 30715A & 0.17 & 43.25 & 79.31 & 264.02 & 489.86 & 56.25 & 314.80 & 68.08 \\
\hline $30744 \mathrm{~A}$ & 2.73 & 26.07 & 44.80 & 196.78 & 381.71 & 36.22 & 242.41 & 52.28 \\
\hline $30725 \mathrm{~A}$ & 0.02 & 9.78 & 9.48 & 96.72 & 228.82 & 18.19 & 143.27 & 38.30 \\
\hline $30750 \mathrm{~A}$ & 0.11 & 18.38 & 49.19 & 126.04 & 269.15 & 24.66 & 175.49 & 48.44 \\
\hline $30762 \mathrm{~A}$ & 0.03 & 48.23 & 65.19 & 272.97 & 515.31 & 60.01 & 336.45 & 90.44 \\
\hline 30773A & 0.03 & 9.48 & 12.90 & 103.91 & 248.28 & 18.53 & 145.89 & 34.31 \\
\hline $30781 \mathrm{~A}$ & 0.03 & 49.71 & 120.01 & 214.89 & 350.14 & 47.94 & 240.44 & 53.28 \\
\hline 30788A & 0.03 & 18.60 & 21.71 & 178.44 & 472.47 & 30.31 & 278.26 & 66.82 \\
\hline 30796A & 0.03 & 38.60 & 77.41 & 226.26 & 441.96 & 49.23 & 291.29 & 85.53 \\
\hline 30803A & 0.03 & 32.23 & 66.76 & 288.61 & 724.17 & 53.02 & 422.83 & 104.65 \\
\hline $30731 \mathrm{~A}$ & 0.96 & 79.60 & 146.77 & 486.79 & 715.96 & 122.90 & 475.84 & 102.72 \\
\hline $30738 \mathrm{~A}$ & 0.06 & 55.96 & 72.56 & 415.16 & 668.33 & 90.28 & 449.25 & 106.72 \\
\hline 30756A & 0.03 & 22.21 & 55.12 & 131.69 & 271.68 & 26.49 & 173.16 & 46.71 \\
\hline $30768 \mathrm{~A}$ & 0.46 & 146.00 & 333.67 & 776.47 & 1043.04 & 211.13 & 735.52 & 169.85 \\
\hline 30778A & 0.03 & 78.39 & 96.55 & 603.24 & 975.47 & 126.93 & 655.42 & 141.87 \\
\hline $30785 \mathrm{~A}$ & 0.03 & 34.30 & 43.13 & 376.14 & 690.70 & 69.41 & 433.57 & 79.97 \\
\hline $30792 \mathrm{~A}$ & 0.03 & 16.18 & 33.67 & 102.65 & 296.24 & 20.21 & 169.25 & 73.79 \\
\hline 30799A & 0.03 & 42.26 & 25.05 & 352.88 & 606.49 & 66.33 & 404.48 & 81.15 \\
\hline $30806 \mathrm{~A}$ & 0.03 & 24.36 & 32.15 & 119.21 & 260.41 & 24.23 & 158.20 & 55.82 \\
\hline
\end{tabular}

104

105 
Table S1 (continued).

\begin{tabular}{|c|c|c|c|c|c|c|c|c|}
\hline Lab ID & PCB128 & PCB180 & PCB170 & PCB195 & PCB206 & PCB209 & Total PCBs & p,p'-DDE \\
\hline $30714 \mathrm{~A}$ & 56.56 & 94.09 & 36.75 & 3.12 & 6.56 & 2.13 & 1576.39 & 118.91 \\
\hline $30722 \mathrm{~A}$ & 136.78 & 161.18 & 75.03 & 5.42 & 10.61 & 3.18 & 3852.38 & 138.78 \\
\hline $30724 \mathrm{~A}$ & 78.00 & 122.78 & 54.27 & 3.53 & 4.75 & 0.50 & 1697.55 & 116.48 \\
\hline 30737A & 49.66 & 73.93 & 31.21 & 2.56 & 4.35 & 1.09 & 1181.11 & 86.35 \\
\hline $30715 A$ & 65.94 & 120.72 & 48.00 & 4.22 & 6.10 & 1.87 & 1599.98 & 178.40 \\
\hline $30744 \mathrm{~A}$ & 49.18 & 79.41 & 31.97 & 3.06 & 4.85 & 1.50 & 1193.99 & 80.52 \\
\hline $30725 \mathrm{~A}$ & 23.30 & 43.75 & 16.83 & 1.04 & 2.47 & 0.02 & 640.26 & 28.94 \\
\hline $30750 \mathrm{~A}$ & 30.47 & 45.58 & 17.54 & 1.66 & 2.93 & 0.59 & 828.87 & 73.03 \\
\hline $30762 \mathrm{~A}$ & 68.01 & 108.05 & 46.38 & 3.20 & 6.22 & 0.43 & 1646.48 & 69.97 \\
\hline 30773A & 24.93 & 48.24 & 17.18 & 0.98 & 2.09 & 0.03 & 673.83 & 45.35 \\
\hline $30781 \mathrm{~A}$ & 51.82 & 56.09 & 23.54 & 1.30 & 2.96 & 0.39 & 1238.11 & 92.02 \\
\hline 30788A & 42.03 & 81.91 & 31.86 & 2.10 & 3.52 & 0.37 & 1238.69 & 41.66 \\
\hline 30796A & 61.71 & 90.68 & 38.80 & 2.61 & 4.94 & 1.02 & 1439.48 & 96.13 \\
\hline 30803A & 69.64 & 127.40 & 47.05 & 3.55 & 5.50 & 0.83 & 1970.77 & 117.90 \\
\hline $30731 \mathrm{~A}$ & 118.42 & 141.46 & 66.24 & 4.49 & 6.83 & 1.19 & 2534.39 & 138.60 \\
\hline 30738A & 107.17 & 130.02 & 61.36 & 3.94 & 5.84 & 1.54 & 2200.96 & 70.68 \\
\hline 30756A & 30.23 & 51.12 & 17.85 & 1.58 & 3.03 & 0.38 & 842.56 & 96.69 \\
\hline 30768A & 193.76 & 245.05 & 118.61 & 8.08 & 9.47 & 1.91 & 4143.75 & 179.19 \\
\hline 30778A & 150.93 & 175.30 & 78.34 & 4.44 & 5.01 & 0.78 & 3134.23 & 101.18 \\
\hline $30785 \mathrm{~A}$ & 80.58 & 105.60 & 41.08 & 2.75 & 4.49 & 0.84 & 1980.99 & 61.94 \\
\hline 30792A & 26.23 & 77.89 & 24.34 & 2.81 & 6.57 & 2.32 & 865.73 & 93.47 \\
\hline 30799A & 71.66 & 100.44 & 41.45 & 2.70 & 4.86 & 1.07 & 1821.84 & 38.20 \\
\hline $30806 \mathrm{~A}$ & 24.89 & 58.24 & 17.98 & 1.86 & 5.07 & 1.99 & 800.26 & 41.48 \\
\hline
\end{tabular}


Table S1 (continued).

\begin{tabular}{|c|c|c|c|c|c|c|c|c|c|}
\hline Lab ID & Sample & Site & Year & Period & Species & PCB008 & PCB018 & PCB028 & PCB052 \\
\hline 30716A & Egg & $\mathrm{BI}$ & 1998 & 1990s & CT & 4.05 & 0.06 & 7.29 & 2.02 \\
\hline 30732A & Egg & $\mathrm{BI}$ & 1998 & 1990s & $\mathrm{CT}$ & 1.13 & 0.07 & 13.76 & 5.49 \\
\hline 30739A & Egg & $\mathrm{BI}$ & 1998 & 1990s & $\mathrm{CT}$ & 0.05 & 0.06 & 66.45 & 19.75 \\
\hline $30745 \mathrm{~A}$ & Egg & $\mathrm{BI}$ & 1998 & 1990s & $\mathrm{CT}$ & 4.07 & 0.07 & 37.86 & 16.37 \\
\hline 30757A & Egg & $\mathrm{BI}$ & 1998 & 1990s & $\mathrm{CT}$ & 1.04 & 0.07 & 13.33 & 3.77 \\
\hline 30763A & Egg & $\mathrm{BI}$ & 1998 & 1990s & $\mathrm{CT}$ & 1.33 & 0.07 & 19.03 & 1.25 \\
\hline 30769A & Egg & $\mathrm{BI}$ & 1998 & 1990s & $\mathrm{CT}$ & 0.33 & 0.07 & 24.23 & 1.13 \\
\hline $30774 \mathrm{~A}$ & Egg & $\mathrm{BI}$ & 1998 & 1990s & $\mathrm{CT}$ & 0.62 & 0.07 & 20.50 & 32.44 \\
\hline $30751 \mathrm{~A}$ & Eggpool & $\mathrm{BI}$ & 1998 & 1990s & $\mathrm{CT}$ & 2.19 & 0.07 & 23.95 & 13.75 \\
\hline $30845 \mathrm{~A}$ & Eggpool & $\mathrm{BI}$ & 1998 & 1990s & $\mathrm{CT}$ & 0.91 & 0.08 & 16.66 & 18.24 \\
\hline 30843A & Eggpool & $\mathrm{BI}$ & 1998 & 1990s & $\mathrm{RT}$ & 0.69 & 0.08 & 19.19 & 8.99 \\
\hline 30846A & Eggpool & $\mathrm{BI}$ & 1998 & 1990s & $\mathrm{RT}$ & 2.61 & 0.05 & 22.45 & 4.13 \\
\hline $30726 A$ & Eggpool & RI & 1998 & $1990 \mathrm{~s}$ & $\mathrm{RT}$ & 1.95 & 0.05 & 18.54 & 3.69 \\
\hline 30718A & Egg & $\mathrm{BI}$ & 1999 & 1990s & $\mathrm{RT}$ & 0.59 & 0.08 & 22.46 & 2.01 \\
\hline 30758A & Egg & $\mathrm{BI}$ & 1999 & 1990s & $\mathrm{RT}$ & 2.77 & 0.08 & 10.57 & 0.13 \\
\hline 30779A & Egg & $\mathrm{BI}$ & 1999 & 1990s & $\mathrm{RT}$ & 3.49 & 0.07 & 19.67 & 1.03 \\
\hline 30790A & Egg & $\mathrm{BI}$ & 1999 & 1990s & $\mathrm{RT}$ & 3.22 & 0.08 & 29.66 & 0.03 \\
\hline $30801 \mathrm{~A}$ & Egg & $\mathrm{BI}$ & 1999 & 1990s & $\mathrm{RT}$ & 1.65 & 0.07 & 4.41 & 0.25 \\
\hline $30810 \mathrm{~A}$ & Egg & $\mathrm{BI}$ & 1999 & 1990s & $\mathrm{RT}$ & 4.73 & 0.08 & 12.10 & 0.03 \\
\hline 30817A & Egg & BI & 1999 & $1990 \mathrm{~s}$ & $\mathrm{RT}$ & 1.85 & 0.08 & 10.35 & 3.02 \\
\hline $30824 \mathrm{~A}$ & Egg & $\mathrm{BI}$ & 1999 & 1990s & RT & 3.77 & 0.08 & 6.50 & 0.32 \\
\hline $30727 \mathrm{~A}$ & Eggpool & BI & 1999 & 1990s & RT & 2.27 & 0.07 & 24.74 & 2.59 \\
\hline
\end{tabular}

111 


\section{Table S1 (continued).}

114

\begin{tabular}{|c|c|c|c|c|c|c|c|c|}
\hline Lab ID & PCB044 & PCB066 & PCB101 & PCB118 & PCB153 & PCB105 & PCB138 & PCB187 \\
\hline $30716 \mathrm{~A}$ & 0.02 & 24.79 & 45.99 & 156.84 & 269.92 & 36.16 & 181.02 & 44.47 \\
\hline 30732A & 0.03 & 33.79 & 83.88 & 179.10 & 322.23 & 39.13 & 213.37 & 54.09 \\
\hline 30739A & 1.27 & 113.10 & 282.07 & 395.76 & 608.59 & 100.55 & 414.70 & 126.47 \\
\hline $30745 \mathrm{~A}$ & 0.64 & 58.54 & 131.03 & 285.28 & 490.13 & 60.98 & 329.03 & 76.48 \\
\hline 30757A & 0.03 & 27.85 & 59.49 & 170.68 & 324.39 & 33.98 & 207.66 & 41.62 \\
\hline $30763 \mathrm{~A}$ & 0.03 & 44.35 & 81.27 & 275.30 & 495.94 & 58.23 & 328.91 & 58.59 \\
\hline 30769A & 0.02 & 57.31 & 103.33 & 303.54 & 594.08 & 67.07 & 393.19 & 106.94 \\
\hline $30774 \mathrm{~A}$ & 1.70 & 56.99 & 173.43 & 299.65 & 526.05 & 67.21 & 358.02 & 86.42 \\
\hline $30751 \mathrm{~A}$ & 0.40 & 49.33 & 114.46 & 287.26 & 531.61 & 55.26 & 338.02 & 70.71 \\
\hline $30845 A$ & 0.71 & 38.29 & 92.21 & 213.04 & 430.53 & 43.60 & 283.22 & 62.26 \\
\hline 30843A & 0.25 & 47.54 & 106.74 & 265.78 & 505.50 & 55.78 & 330.57 & 67.41 \\
\hline 30846A & 0.02 & 47.58 & 99.86 & 239.02 & 415.29 & 56.27 & 290.12 & 85.50 \\
\hline $30726 \mathrm{~A}$ & 0.02 & 40.81 & 77.12 & 235.07 & 419.62 & 53.98 & 280.84 & 76.68 \\
\hline 30718A & 0.03 & 54.45 & 59.89 & 291.10 & 570.80 & 69.22 & 370.20 & 120.12 \\
\hline $30758 \mathrm{~A}$ & 0.03 & 24.77 & 49.25 & 168.73 & 357.88 & 30.54 & 225.10 & 43.28 \\
\hline 30779A & 0.03 & 41.72 & 90.77 & 201.49 & 384.95 & 39.41 & 250.48 & 60.59 \\
\hline 30790A & 0.03 & 56.60 & 92.43 & 345.17 & 686.81 & 65.71 & 423.65 & 94.71 \\
\hline 30801A & 0.03 & 21.55 & 16.93 & 238.89 & 513.10 & 41.17 & 323.95 & 83.10 \\
\hline $30810 A$ & 0.03 & 22.31 & 35.83 & 153.89 & 315.55 & 25.53 & 201.93 & 47.15 \\
\hline 30817A & 0.03 & 33.20 & 72.86 & 265.31 & 495.10 & 53.22 & 323.66 & 90.07 \\
\hline $30824 \mathrm{~A}$ & 0.03 & 17.31 & 32.27 & 131.82 & 329.90 & 24.48 & 203.24 & 43.90 \\
\hline $30727 \mathrm{~A}$ & 0.03 & 57.28 & 91.04 & 346.60 & 618.76 & 70.15 & 406.06 & 114.94 \\
\hline
\end{tabular}

115

116 


\section{Table S1 (continued).}

118

\begin{tabular}{|c|c|c|c|c|c|c|c|c|}
\hline Lab ID & PCB128 & PCB180 & PCB170 & PCB195 & PCB206 & PCB209 & Total PCBs & p,p'-DDE \\
\hline 30716A & 40.72 & 53.18 & 22.55 & 1.57 & 3.37 & 0.88 & 894.88 & 77.95 \\
\hline 30732A & 42.80 & 61.42 & 23.78 & 1.86 & 4.02 & 1.50 & 1081.43 & 141.12 \\
\hline 30739A & 101.20 & 124.62 & 54.06 & 4.46 & 7.92 & 2.44 & 2423.53 & 212.89 \\
\hline 30745A & 75.36 & 102.28 & 43.22 & 3.44 & 6.43 & 2.04 & 1723.24 & 112.42 \\
\hline 30757A & 40.45 & 63.35 & 23.09 & 1.80 & 4.54 & 1.20 & 1018.32 & 95.87 \\
\hline $30763 \mathrm{~A}$ & 66.97 & 98.16 & 41.65 & 2.93 & 4.34 & 1.01 & 1579.37 & 150.86 \\
\hline 30769A & 86.97 & 163.99 & 71.33 & 5.81 & 6.79 & 1.27 & 1987.41 & 173.43 \\
\hline 30774A & 72.84 & 90.92 & 34.90 & 2.64 & 3.80 & 0.59 & 1828.78 & 145.99 \\
\hline $30751 \mathrm{~A}$ & 68.43 & 93.16 & 35.78 & 3.06 & 4.98 & 1.72 & 1694.13 & 145.26 \\
\hline 30845A & 50.51 & 70.38 & 28.36 & 2.09 & 3.25 & 0.63 & 1354.97 & 124.23 \\
\hline 30843A & 64.74 & 95.66 & 37.11 & 2.78 & 4.17 & 0.83 & 1613.82 & 146.02 \\
\hline 30846A & 64.40 & 95.31 & 40.43 & 3.42 & 6.10 & 1.92 & 1474.48 & 101.54 \\
\hline 30726A & 61.82 & 85.49 & 38.70 & 2.77 & 5.20 & 1.00 & 1403.36 & 72.31 \\
\hline 30718A & 80.91 & 133.28 & 54.54 & 4.61 & 8.73 & 2.40 & 1845.40 & 85.39 \\
\hline 30758A & 41.15 & 57.72 & 22.77 & 1.37 & 3.11 & 0.37 & 1039.60 & 45.90 \\
\hline 30779A & 49.11 & 68.70 & 27.42 & 1.82 & 4.43 & 1.17 & 1246.36 & 88.54 \\
\hline $30790 \mathrm{~A}$ & 87.44 & 132.59 & 52.47 & 3.40 & 5.50 & 1.18 & 2080.66 & 89.75 \\
\hline 30801A & 56.39 & 92.27 & 36.61 & 2.60 & 4.92 & 1.33 & 1439.22 & 27.09 \\
\hline $30810 \mathrm{~A}$ & 37.34 & 53.63 & 21.30 & 1.30 & 3.01 & 0.57 & 936.30 & 59.04 \\
\hline 30817A & 71.31 & 99.60 & 43.38 & 2.90 & 5.03 & 1.28 & 1572.26 & 67.58 \\
\hline 30824A & 35.39 & 64.73 & 24.65 & 1.78 & 4.22 & 0.74 & 925.13 & 55.71 \\
\hline 30727A & 91.32 & 133.99 & 56.56 & 4.40 & 7.81 & 1.15 & 2029.77 & 96.22 \\
\hline
\end{tabular}




\section{Table S1 (continued).}

122

\begin{tabular}{|l|r|r|r|r|r|r|r|r|r|}
\hline Lab ID & Sample & Site & Year & Period & Species & PCB008 & PCB018 & PCB028 & PCB052 \\
\hline 30733A & Egg & RI & 1999 & $1990 s$ & CT & 1.15 & $\mathbf{0 . 0 6}$ & 44.50 & 2.37 \\
\hline 30770A & Egg & RI & 1999 & $1990 s$ & CT & $\mathbf{0 . 0 4}$ & $\mathbf{0 . 0 6}$ & 31.24 & 1.13 \\
\hline 30782A & Egg & RI & 1999 & $1990 s$ & CT & $\mathbf{0 . 0 5}$ & $\mathbf{0 . 0 6}$ & 127.59 & 233.31 \\
\hline 30793A & Egg & RI & 1999 & $1990 s$ & CT & $\mathbf{0 . 0 5}$ & $\mathbf{0 . 0 6}$ & 53.00 & 39.09 \\
\hline 30804A & Egg & RI & 1999 & $1990 s$ & CT & $\mathbf{0 . 0 6}$ & $\mathbf{0 . 0 8}$ & 17.46 & 0.35 \\
\hline 30812A & Egg & RI & 1999 & $1990 s$ & CT & 0.66 & $\mathbf{0 . 0 6}$ & 14.04 & 17.26 \\
\hline 30820A & Egg & RI & 1999 & $1990 s$ & CT & 0.90 & $\mathbf{0 . 0 8}$ & 15.20 & 16.74 \\
\hline 30827A & Egg & RI & 1999 & $1990 s$ & CT & $\mathbf{0 . 0 5}$ & $\mathbf{0 . 0 7}$ & 22.90 & 8.26 \\
\hline 30764A & Eggpool & RI & 1999 & $1990 s$ & CT & 1.07 & $\mathbf{0 . 0 7}$ & 16.03 & 3.48 \\
\hline 30746A & Egg & RI & 1999 & $1990 s$ & RT & 1.19 & $\mathbf{0 . 0 8}$ & 7.29 & 0.49 \\
\hline 30775A & Egg & RI & 1999 & $1990 s$ & RT & 1.12 & $\mathbf{0 . 0 7}$ & 8.30 & 1.50 \\
\hline 30786A & Egg & RI & 1999 & $1990 s$ & RT & 0.31 & $\mathbf{0 . 0 8}$ & 43.14 & 5.38 \\
\hline 30797A & Egg & RI & 1999 & $1990 s$ & RT & $\mathbf{0 . 0 6}$ & $\mathbf{0 . 0 8}$ & 39.53 & 2.82 \\
\hline 30808A & Egg & RI & 1999 & $1990 s$ & RT & 3.20 & $\mathbf{0 . 0 7}$ & 23.28 & 1.79 \\
\hline 30815A & Egg & RI & 1999 & $1990 s$ & RT & $\mathbf{0 . 0 6}$ & $\mathbf{0 . 0 8}$ & 17.87 & 0.79 \\
\hline 30822A & Egg & RI & 1999 & $1990 s$ & RT & 1.18 & $\mathbf{0 . 0 7}$ & 9.52 & 0.61 \\
\hline 30829A & Egg & RI & 1999 & $1990 s$ & RT & 1.13 & $\mathbf{0 . 0 7}$ & 13.58 & 0.70 \\
\hline 30740A & Eggpool & RI & 1999 & $1990 s$ & RT & 2.42 & $\mathbf{0 . 0 7}$ & 17.33 & 1.64 \\
\hline 30752A & Eggpool & RI & 1999 & $1990 s$ & RT & 0.78 & $\mathbf{0 . 0 7}$ & 17.60 & 1.63 \\
\hline
\end{tabular}

123 
125

126

\begin{tabular}{|l|r|r|r|r|r|r|r|r|}
\hline Lab ID & PCB044 & PCB066 & PCB101 & PCB118 & PCB153 & PCB105 & PCB138 & PCB187 \\
\hline 30733A & $\mathbf{0 . 0 2}$ & 171.25 & 151.88 & 872.20 & 1200.37 & 220.37 & 823.69 & 192.62 \\
\hline 30770A & $\mathbf{0 . 0 2}$ & 77.02 & 78.25 & 419.61 & 751.33 & 101.63 & 514.03 & 172.45 \\
\hline 30782A & 23.49 & 137.40 & 331.80 & 391.97 & 502.67 & 112.89 & 366.11 & 106.94 \\
\hline 30793A & 0.98 & 127.48 & 282.53 & 696.39 & 1162.25 & 141.43 & 767.30 & 221.03 \\
\hline 30804A & $\mathbf{0 . 0 3}$ & 36.86 & 68.90 & 236.20 & 484.36 & 44.65 & 294.13 & 54.73 \\
\hline 30812A & 0.06 & 32.06 & 81.50 & 253.67 & 494.23 & 48.38 & 322.83 & 81.24 \\
\hline 30820A & 0.85 & 36.08 & 104.41 & 253.01 & 533.77 & 45.99 & 326.25 & 74.57 \\
\hline 30827A & $\mathbf{0 . 0 3}$ & 62.59 & 131.57 & 266.20 & 444.67 & 73.79 & 315.38 & 125.75 \\
\hline 30764A & $\mathbf{0 . 0 3}$ & 42.18 & 78.43 & 277.78 & 498.02 & 58.47 & 328.33 & 90.97 \\
\hline 30746A & $\mathbf{0 . 0 3}$ & 14.79 & 39.07 & 80.93 & 158.82 & 17.42 & 104.23 & 28.80 \\
\hline 30775A & $\mathbf{0 . 0 3}$ & 25.19 & 52.87 & 240.42 & 497.42 & 42.14 & 309.45 & 76.34 \\
\hline 30786A & $\mathbf{0 . 0 3}$ & 89.28 & 223.43 & 386.48 & 660.89 & 84.26 & 429.54 & 108.06 \\
\hline 30797A & $\mathbf{0 . 0 3}$ & 82.18 & 90.40 & 454.61 & 718.22 & 101.40 & 497.07 & 132.99 \\
\hline 30808A & $\mathbf{0 . 0 3}$ & 54.46 & 99.51 & 287.49 & 558.77 & 56.83 & 355.47 & 84.43 \\
\hline 30815A & $\mathbf{0 . 0 3}$ & 36.67 & 30.50 & 189.51 & 352.39 & 40.60 & 232.56 & 58.11 \\
\hline 30822A & $\mathbf{0 . 0 3}$ & 20.72 & 51.42 & 116.14 & 257.95 & 26.05 & 164.86 & 49.84 \\
\hline 30829A & $\mathbf{0 . 0 2}$ & 35.20 & 45.50 & 152.37 & 258.84 & 38.46 & 183.94 & 58.54 \\
\hline 30740A & $\mathbf{0 . 0 2}$ & 34.78 & 53.92 & 218.92 & 433.75 & 45.40 & 274.31 & 74.54 \\
\hline 30752A & $\mathbf{0 . 0 2}$ & 45.44 & 69.22 & 293.50 & 536.72 & 60.39 & 352.38 & 90.24 \\
\hline
\end{tabular}

127

128

Table S1 (continued). 
Table S1 (continued).

130

\begin{tabular}{|l|r|r|r|r|r|r|r|r|}
\hline Lab ID & PCB128 & PCB180 & PCB170 & PCB195 & PCB206 & PCB209 & Total PCBs & p,p'-DDE \\
\hline 30733A & 247.20 & 274.27 & 141.81 & 10.04 & 11.35 & 2.27 & 4367.41 & 153.41 \\
\hline 30770A & 126.68 & 177.93 & 85.67 & 6.44 & 8.79 & 1.59 & 2553.92 & 116.74 \\
\hline 30782A & 103.40 & 112.10 & 61.03 & 4.21 & 6.23 & 1.31 & 2622.56 & 107.56 \\
\hline 30793A & 170.82 & 253.28 & 101.08 & 9.76 & 16.84 & 5.62 & 4048.97 & 168.01 \\
\hline 30804A & 52.71 & 79.26 & 30.19 & 2.26 & 4.14 & 1.10 & 1407.45 & 90.55 \\
\hline 30812A & 69.14 & 115.36 & 49.29 & 3.64 & 4.44 & 0.93 & 1588.78 & 99.86 \\
\hline 30820A & 64.41 & 105.02 & 41.78 & 4.20 & 11.68 & 5.74 & 1640.67 & 64.67 \\
\hline 30827A & 72.97 & 151.59 & 66.79 & 7.93 & 20.11 & 6.27 & 1776.91 & 162.17 \\
\hline 30764A & 70.37 & 113.77 & 50.00 & 4.28 & 9.46 & 3.51 & 1646.24 & 109.51 \\
\hline 30746A & 19.32 & 30.86 & 11.29 & 0.72 & 1.66 & 0.02 & 517.01 & 62.58 \\
\hline 30775A & 60.23 & 88.82 & 37.07 & 2.68 & 5.09 & 1.50 & 1450.24 & 75.86 \\
\hline 30786A & 92.96 & 98.03 & 39.92 & 2.73 & 4.91 & 1.30 & 2270.75 & 145.33 \\
\hline 30797A & 117.58 & 135.72 & 65.23 & 3.93 & 6.66 & 1.56 & 2450.08 & 81.96 \\
\hline 30808A & 67.91 & 96.47 & 35.02 & 3.34 & 6.36 & 2.45 & 1736.86 & 94.28 \\
\hline 30815A & 44.49 & 56.75 & 24.82 & 1.55 & 4.12 & 0.56 & 1091.46 & 42.20 \\
\hline 30822A & 29.72 & 53.28 & 19.47 & 1.68 & 3.15 & 0.39 & 806.09 & 73.77 \\
\hline 30829A & 39.71 & 53.71 & 23.24 & 1.63 & 3.49 & 0.47 & 910.59 & 68.28 \\
\hline 30740A & 58.40 & 87.35 & 37.78 & 2.87 & 5.35 & 1.46 & 1350.30 & 69.58 \\
\hline 30752A & 76.25 & 103.40 & 45.40 & 3.32 & 6.32 & 2.05 & 1704.74 & 74.44 \\
\hline
\end{tabular}

131 
Table S1 (continued).

134

\begin{tabular}{|l|r|r|r|r|r|r|r|r|r|}
\hline Lab ID & Sample & Site & Year & Period & Species & PCB008 & PCB018 & PCB028 & PCB052 \\
\hline 30760A & Egg & BI & 2005 & 2000s & CT & $\mathbf{0 . 0 7}$ & $\mathbf{0 . 1 8}$ & 19.15 & 57.76 \\
\hline 30791A & Egg & BI & 2005 & 2000s & CT & $\mathbf{0 . 0 7}$ & $\mathbf{0 . 0 9}$ & 20.10 & 3.41 \\
\hline 30805A & Egg & BI & 2005 & 2000s & CT & 0.23 & $\mathbf{0 . 0 8}$ & 24.72 & 0.03 \\
\hline 30816A & Egg & BI & 2005 & 2000s & CT & $\mathbf{0 . 0 7}$ & $\mathbf{0 . 0 9}$ & 19.15 & 1.53 \\
\hline 30826A & Egg & BI & 2005 & 2000s & CT & 0.71 & $\mathbf{0 . 0 8}$ & 10.82 & 3.12 \\
\hline 30833A & Egg & BI & 2005 & 2000s & CT & 1.27 & $\mathbf{0 . 0 8}$ & 11.88 & 0.80 \\
\hline 30838A & Egg & BI & 2005 & 2000s & CT & $\mathbf{0 . 0 6}$ & $\mathbf{0 . 0 8}$ & 10.65 & 9.31 \\
\hline 30844A & Egg & BI & 2005 & 2000s & CT & 0.19 & $\mathbf{0 . 0 8}$ & 5.93 & 0.31 \\
\hline 30748A & Egg & BI & 2005 & 2000s & RT & 0.96 & $\mathbf{0 . 0 8}$ & 2.98 & 0.61 \\
\hline 30787A & Egg & BI & 2005 & 2000s & RT & 0.35 & $\mathbf{0 . 0 8}$ & 7.38 & 0.03 \\
\hline 30802A & Egg & BI & 2005 & 2000s & RT & $\mathbf{0 . 0 6}$ & $\mathbf{0 . 0 8}$ & 5.96 & 0.04 \\
\hline 30814A & Egg & BI & 2005 & 2000s & RT & 1.21 & $\mathbf{0 . 0 8}$ & 4.90 & 0.03 \\
\hline 30823A & Egg & BI & 2005 & 2000s & RT & $\mathbf{0 . 0 6}$ & $\mathbf{0 . 0 8}$ & 5.49 & 0.03 \\
\hline 30832A & Egg & BI & 2005 & 2000s & RT & 0.34 & $\mathbf{0 . 0 8}$ & 8.25 & 0.03 \\
\hline 30836A & Egg & BI & 2005 & 2000s & RT & 0.33 & $\mathbf{0 . 0 8}$ & 5.00 & 0.03 \\
\hline 30841A & Egg & BI & 2005 & 2000s & RT & 0.71 & $\mathbf{0 . 0 9}$ & 9.21 & 0.04 \\
\hline 30784A & Eggpool & BI & 2005 & 2000s & RT & 0.43 & $\mathbf{0 . 0 9}$ & 9.99 & 0.61 \\
\hline
\end{tabular}


Table S1 (continued).

138

\begin{tabular}{|l|r|r|r|r|r|r|r|r|}
\hline Lab ID & PCB044 & PCB066 & PCB101 & PCB118 & PCB153 & PCB105 & PCB138 & PCB187 \\
\hline 30760A & 11.95 & 39.95 & 171.62 & 282.89 & 535.25 & 58.55 & 337.88 & 110.82 \\
\hline 30791A & $\mathbf{0 . 0 3}$ & 48.95 & 67.42 & 291.50 & 709.08 & 65.74 & 478.68 & 163.48 \\
\hline 30805A & $\mathbf{0 . 0 3}$ & 29.62 & 22.86 & 158.36 & 363.39 & 31.57 & 214.55 & 35.62 \\
\hline 30816A & $\mathbf{0 . 0 3}$ & 69.50 & 73.63 & 402.50 & 683.03 & 87.52 & 442.32 & 115.02 \\
\hline 30826A & $\mathbf{0 . 0 3}$ & 21.66 & 52.74 & 122.51 & 273.30 & 24.77 & 171.73 & 53.75 \\
\hline 30833A & $\mathbf{0 . 0 3}$ & 28.55 & 31.88 & 164.81 & 377.69 & 37.90 & 254.08 & 79.54 \\
\hline 30838A & $\mathbf{0 . 0 3}$ & 23.14 & 49.24 & 147.47 & 335.01 & 28.96 & 208.97 & 50.46 \\
\hline 30844A & $\mathbf{0 . 0 3}$ & 18.51 & 28.39 & 137.56 & 314.24 & 28.20 & 207.93 & 67.16 \\
\hline 30748A & $\mathbf{0 . 0 3}$ & 12.54 & 18.02 & 123.21 & 316.54 & 20.94 & 187.76 & 57.04 \\
\hline 30787A & $\mathbf{0 . 0 3}$ & 20.41 & 29.75 & 180.25 & 442.72 & 33.12 & 268.60 & 75.06 \\
\hline 30802A & $\mathbf{0 . 0 3}$ & 20.06 & 15.99 & 176.73 & 412.13 & 30.77 & 249.82 & 68.26 \\
\hline 30814A & $\mathbf{0 . 0 3}$ & 16.61 & 19.54 & 142.71 & 322.74 & 27.02 & 200.47 & 61.43 \\
\hline 30823A & $\mathbf{0 . 0 3}$ & 11.46 & 11.64 & 94.40 & 249.79 & 17.14 & 146.57 & 38.37 \\
\hline 30832A & $\mathbf{0 . 0 3}$ & 18.69 & 17.10 & 156.63 & 400.52 & 26.79 & 246.08 & 65.02 \\
\hline 30836A & $\mathbf{0 . 0 3}$ & 14.07 & 12.72 & 138.33 & 382.13 & 25.24 & 230.93 & 84.71 \\
\hline 30841A & $\mathbf{0 . 0 3}$ & 21.55 & 30.94 & 172.65 & 414.12 & 31.59 & 256.35 & 85.67 \\
\hline 30784A & $\mathbf{0 . 0 3}$ & 23.24 & 45.77 & 165.13 & 408.15 & 30.55 & 237.73 & 71.11 \\
\hline
\end{tabular}




\section{Table S1 (continued).}

142

\begin{tabular}{|l|r|r|r|r|r|r|r|r|}
\hline Lab ID & PCB128 & PCB180 & PCB170 & PCB195 & PCB206 & PCB209 & Total PCBs & p,p'-DDE \\
\hline 30760A & 70.62 & 119.88 & 46.62 & 4.34 & 8.33 & 2.16 & 1878.02 & 143.03 \\
\hline 30791A & 81.30 & 338.39 & 136.58 & 17.91 & 10.10 & 0.98 & 2433.80 & 195.62 \\
\hline 30805A & 38.54 & 78.55 & 28.03 & 2.28 & 5.76 & 2.09 & 1036.31 & 92.64 \\
\hline 30816A & 103.96 & 146.96 & 62.01 & 5.60 & 11.02 & 2.82 & 2226.77 & 152.80 \\
\hline 30826A & 31.03 & 61.72 & 22.27 & 2.15 & 5.62 & 1.95 & 859.97 & 75.94 \\
\hline 30833A & 40.54 & 137.02 & 50.93 & 5.89 & 9.02 & 2.74 & 1234.65 & 120.48 \\
\hline 30838A & 37.23 & 64.07 & 24.07 & 1.91 & 2.59 & 0.47 & 993.74 & 123.72 \\
\hline 30844A & 35.53 & 82.90 & 30.38 & 3.94 & 9.85 & 4.77 & 975.88 & 76.77 \\
\hline 30748A & 31.81 & 64.88 & 23.59 & 2.78 & 9.50 & 5.61 & 878.85 & 45.06 \\
\hline 30787A & 47.80 & 83.60 & 32.71 & 2.99 & 7.94 & 3.49 & 1236.30 & 69.82 \\
\hline 30802A & 41.82 & 72.94 & 27.70 & 2.21 & 5.49 & 2.10 & 1132.19 & 29.93 \\
\hline 30814A & 37.23 & 76.89 & 28.53 & 3.04 & 9.64 & 4.48 & 956.58 & 55.14 \\
\hline 30823A & 23.03 & 43.10 & 15.69 & 1.08 & 3.14 & 0.51 & 661.61 & 24.67 \\
\hline 30832A & 37.12 & 73.51 & 28.11 & 2.49 & 6.69 & 2.50 & 1089.99 & 36.74 \\
\hline 30836A & 35.82 & 95.06 & 32.58 & 3.89 & 13.21 & 6.33 & 1080.51 & 34.55 \\
\hline 30841A & 45.16 & 94.37 & 36.83 & 3.89 & 9.05 & 3.35 & 1215.59 & 77.79 \\
\hline 30784A & 41.84 & 71.37 & 26.66 & 2.56 & 6.87 & 3.33 & 1145.43 & 64.54 \\
\hline
\end{tabular}

143

144 
Table S1 (continued).

146

\begin{tabular}{|l|r|r|r|r|r|r|r|r|r|}
\hline Lab ID & Sample & Site & Year & Period & Species & PCB008 & PCB018 & PCB028 & PCB052 \\
\hline 30734A & Egg & RI & 2005 & 2000s & CT & 0.93 & $\mathbf{0 . 0 8}$ & 6.21 & 0.44 \\
\hline 30780A & Egg & RI & 2005 & 2000s & CT & 0.67 & $\mathbf{0 . 0 9}$ & 44.99 & 1.05 \\
\hline 30798A & Egg & RI & 2005 & 2000s & CT & $\mathbf{0 . 0 6}$ & $\mathbf{0 . 0 8}$ & 198.51 & 19.65 \\
\hline 30811A & Egg & RI & 2005 & 2000s & CT & 0.59 & $\mathbf{0 . 0 7}$ & 5.73 & 0.03 \\
\hline 30821A & Egg & RI & 2005 & 2000s & CT & $\mathbf{0 . 0 6}$ & $\mathbf{0 . 0 8}$ & 15.84 & 3.76 \\
\hline 30830A & Egg & RI & 2005 & 2000s & CT & $\mathbf{0 . 0 6}$ & $\mathbf{0 . 0 8}$ & 150.98 & 12.16 \\
\hline 30835A & Egg & RI & 2005 & 2000s & CT & 1.32 & $\mathbf{0 . 0 8}$ & 8.48 & 4.21 \\
\hline 30840A & Egg & RI & 2005 & 2000s & CT & $\mathbf{0 . 0 6}$ & $\mathbf{0 . 0 8}$ & 7.17 & 0.03 \\
\hline 30719A & Egg & RI & 2005 & 2000s & RT & 0.38 & $\mathbf{0 . 0 8}$ & 2.79 & 0.50 \\
\hline 30772A & Egg & RI & 2005 & 2000s & RT & $\mathbf{0 . 0 7}$ & $\mathbf{0 . 1 0}$ & 5.59 & 0.41 \\
\hline 30794A & Egg & RI & 2005 & 2000s & RT & $\mathbf{0 . 0 6}$ & $\mathbf{0 . 0 8}$ & 3.10 & 0.89 \\
\hline 30809A & Egg & RI & 2005 & 2000s & RT & 0.43 & $\mathbf{0 . 0 8}$ & 7.28 & 0.81 \\
\hline 30818A & Egg & RI & 2005 & 2000s & RT & $\mathbf{0 . 0 6}$ & $\mathbf{0 . 0 8}$ & 3.20 & 0.34 \\
\hline 30828A & Egg & RI & 2005 & 2000s & RT & $\mathbf{0 . 0 6}$ & $\mathbf{0 . 0 8}$ & 7.59 & 0.19 \\
\hline 30834A & Egg & RI & 2005 & 2000s & RT & 1.71 & $\mathbf{0 . 0 8}$ & 7.17 & 0.03 \\
\hline 30839A & Egg & RI & 2005 & 2000s & RT & 0.82 & $\mathbf{0 . 0 8}$ & 2.01 & 0.03 \\
\hline 30776A & Eggpool & RI & 2005 & 2000s & RT & 0.77 & $\mathbf{0 . 0 7}$ & 10.63 & 3.21 \\
\hline
\end{tabular}

147 
Table S1 (continued).

150

\begin{tabular}{|l|r|r|r|r|r|r|r|r|}
\hline Lab ID & PCB044 & PCB066 & PCB101 & PCB118 & PCB153 & PCB105 & PCB138 & PCB187 \\
\hline 30734A & $\mathbf{0 . 0 3}$ & 14.36 & 21.62 & 110.33 & 284.41 & 20.61 & 157.05 & 32.08 \\
\hline 30780A & $\mathbf{0 . 0 3}$ & 57.37 & 65.59 & 272.32 & 480.50 & 57.62 & 300.74 & 64.52 \\
\hline 30798A & $\mathbf{0 . 0 3}$ & 146.30 & 303.28 & 556.06 & 803.12 & 122.51 & 501.30 & 111.92 \\
\hline 30811A & $\mathbf{0 . 0 3}$ & 12.32 & 17.77 & 100.40 & 274.20 & 17.86 & 145.43 & 32.15 \\
\hline 30821A & $\mathbf{0 . 0 3}$ & 34.02 & 58.14 & 208.14 & 432.39 & 40.63 & 258.51 & 62.84 \\
\hline 30830A & $\mathbf{0 . 0 3}$ & 117.11 & 227.94 & 444.84 & 667.50 & 102.54 & 440.59 & 98.78 \\
\hline 30835A & $\mathbf{0 . 0 3}$ & 20.90 & 52.62 & 95.46 & 221.84 & 22.11 & 146.30 & 59.39 \\
\hline 30840A & $\mathbf{0 . 0 3}$ & 15.97 & 20.55 & 121.02 & 296.33 & 21.99 & 173.06 & 37.36 \\
\hline 30719A & $\mathbf{0 . 0 3}$ & 8.57 & 19.31 & 92.54 & 216.73 & 15.85 & 126.78 & 36.08 \\
\hline 30772A & $\mathbf{0 . 0 3}$ & 16.62 & 28.83 & 164.29 & 347.87 & 30.36 & 217.65 & 54.16 \\
\hline 30794A & $\mathbf{0 . 0 3}$ & 11.48 & 19.77 & 116.40 & 350.14 & 20.35 & 200.40 & 72.85 \\
\hline 30809A & $\mathbf{0 . 0 3}$ & 25.71 & 37.48 & 250.53 & 554.96 & 44.04 & 335.49 & 118.81 \\
\hline 30818A & $\mathbf{0 . 0 3}$ & 8.25 & 12.50 & 68.64 & 200.20 & 12.54 & 118.54 & 41.47 \\
\hline 30828A & $\mathbf{0 . 0 3}$ & 27.58 & 14.04 & 189.09 & 377.72 & 33.91 & 236.01 & 57.18 \\
\hline 30834A & $\mathbf{0 . 0 3}$ & 19.11 & 32.91 & 126.02 & 309.72 & 24.32 & 193.09 & 65.20 \\
\hline 30839A & $\mathbf{0 . 0 3}$ & 8.09 & 10.93 & 98.17 & 257.67 & 17.53 & 158.48 & 36.96 \\
\hline 30776A & $\mathbf{0 . 0 3}$ & 27.89 & 48.02 & 190.15 & 430.75 & 37.12 & 260.10 & 86.73 \\
\hline
\end{tabular}

151

152 


\section{Table S1 (continued).}

154

\begin{tabular}{|l|r|r|r|r|r|r|r|r|}
\hline Lab ID & PCB128 & PCB180 & PCB170 & PCB195 & PCB206 & PCB209 & Total PCBs & p,p'-DDE \\
\hline 30734A & 28.77 & 65.71 & 24.52 & 2.15 & 4.43 & 0.79 & 774.50 & 60.49 \\
\hline 30780A & 68.31 & 101.93 & 42.33 & 3.34 & 7.98 & 2.43 & 1571.79 & 99.44 \\
\hline 30798A & 118.68 & 149.04 & 64.51 & 4.31 & 9.17 & 3.23 & 3111.75 & 78.31 \\
\hline 30811A & 25.20 & 69.21 & 24.72 & 2.44 & 5.41 & 1.56 & 735.12 & 53.29 \\
\hline 30821A & 49.14 & 88.07 & 33.74 & 3.64 & 15.21 & 6.83 & 1311.09 & 59.84 \\
\hline 30830A & 103.79 & 143.83 & 63.96 & 5.06 & 13.63 & 4.94 & 2597.81 & 68.00 \\
\hline 30835A & 23.52 & 46.16 & 14.42 & 1.95 & 5.80 & 2.32 & 726.90 & 125.08 \\
\hline 30840A & 29.94 & 61.24 & 22.56 & 1.95 & 8.57 & 2.39 & 820.29 & 51.35 \\
\hline 30719A & 22.10 & 42.64 & 15.26 & 1.37 & 4.27 & 1.66 & 606.95 & 49.86 \\
\hline 30772A & 42.32 & 62.41 & 25.83 & 1.62 & 5.57 & 1.67 & 1005.42 & 54.98 \\
\hline 30794A & 30.79 & 77.68 & 27.32 & 3.50 & 11.32 & 5.87 & 952.02 & 25.48 \\
\hline 30809A & 63.77 & 133.84 & 46.94 & 6.15 & 27.51 & 15.71 & 1669.56 & 84.50 \\
\hline 30818A & 17.89 & 48.26 & 16.35 & 1.78 & 8.95 & 5.07 & 564.15 & 17.60 \\
\hline 30828A & 38.12 & 71.20 & 28.28 & 2.45 & 7.73 & 3.52 & 1094.79 & 18.51 \\
\hline 30834A & 33.23 & 67.53 & 22.81 & 2.60 & 7.58 & 3.01 & 916.17 & 64.14 \\
\hline 30839A & 27.60 & 54.84 & 20.98 & 1.82 & 6.40 & 2.83 & 705.26 & 37.92 \\
\hline 30776A & 49.30 & 90.25 & 34.79 & 3.61 & 10.67 & 4.90 & 1288.99 & 84.48 \\
\hline
\end{tabular}


156 Table S2: PCB congeners in New Bedford Harbor upper sediment cores (ng g ${ }^{-1}$ dry weight) collected, dated and analyzed as described [15], where values shown in bold are $1 / 2$ minimum detection limit (MDL). (**Values for 1996 are presented as the average of three

158 core slices dated to that year.)

\begin{tabular}{|c|c|c|c|c|c|c|c|c|c|c|c|c|c|c|c|c|c|c|c|c|}
\hline ID & $\begin{array}{c}\text { Date, } \\
\text { Calculated* }\end{array}$ & РCB008 & РСB018 & РСВ028 & РСB052 & РСВ044 & РCB066 & РCB101 & PCB118 & PCB153 & PCB105 & PCB138 & РСB187 & PCB128 & PCB180 & PCB170 & PCB195 & PCB206 & РCB209 & $\begin{array}{l}\text { Sum } \\
\text { PCBs }^{*}\end{array}$ \\
\hline $\mathrm{NBH} 1 \mathrm{a}$ & $1996^{\star \star}$ & 1211 & 2985 & 9997 & 7074 & 4319 & 3517 & 4023 & 4329 & 3704 & 1327 & 2852 & 540 & 517 & 620 & 353 & 50 & 62 & 19 & 47499 \\
\hline $\mathrm{NBH} 1 \mathrm{a}$ & 1983 & 4820 & 9930 & 28400 & 16800 & 12400 & 7040 & 7260 & 6900 & 5780 & 2380 & 4860 & 866 & 938 & 1000 & 606 & 91.5 & 99.2 & 29.2 & 110200 \\
\hline $\mathrm{NBH} 1 \mathrm{a}$ & 1975 & 8300 & 16100 & 45200 & 23800 & 19200 & 15100 & 12900 & 12100 & 8710 & 3370 & 6940 & 1210 & 1320 & 1520 & 884 & 122 & 136 & 28.6 & 176941 \\
\hline NBH1a & 1971 & 7650 & 14500 & 46600 & 21700 & 18200 & 15000 & 12500 & 12200 & 8300 & 2940 & 6380 & 1100 & 1140 & 1530 & 902 & 113 & 137 & 34.7 & 170927 \\
\hline $\mathrm{NBH} 1 \mathrm{a}$ & 1966 & 6865 & 13750 & 43100 & 21650 & 18500 & 21600 & 16950 & 16850 & 10650 & 2830 & 7425 & 1435 & 1205 & 1845 & 1070 & 138.5 & 150.5 & 36.5 & 186051 \\
\hline $\mathrm{NBH} 1 \mathrm{a}$ & 1962 & 5100 & 9240 & 31500 & 13600 & 12400 & 17900 & 12000 & 13100 & 7450 & 2360 & 4970 & 886 & 827 & 1300 & 821 & 87.5 & 108 & 24.8 & 133674 \\
\hline $\mathrm{NBH} 1 \mathrm{a}$ & 1954 & 1700 & 3390 & 9320 & 5480 & 4560 & 7510 & 6560 & 7510 & 4890 & 1430 & 3030 & 602 & 484 & 866 & 499 & 69.8 & 74.2 & 25.2 & 58000 \\
\hline NBH1a & 1941 & 960 & 1865 & 6280 & 2775 & 2475 & 4520 & 3360 & 3975 & 2240 & 632.5 & 1610 & 251.5 & 331 & 415.5 & 262 & 34.15 & 51.8 & 22.15 & 32061 \\
\hline $\mathrm{NBH} 1 \mathrm{a}$ & 1937 & 556 & 1060 & 3490 & 1620 & 1380 & 2740 & 2360 & 2830 & 1610 & 427 & 1220 & 167 & 256 & 288 & 186 & 22.3 & 34.9 & 14.8 & 20262 \\
\hline NBH1a & 1924 & 33.9 & 57.2 & 130 & 117 & 72.1 & 140 & 214 & 248 & 153 & 75.9 & 146 & 15.4 & 33.0 & 26.0 & 15.7 & 2.29 & 3.12 & 2.01 & 1485 \\
\hline $\mathrm{NBH} 1 \mathrm{a}$ & 1907 & 1.1 & 2.1 & 3.6 & 4.2 & 0.9 & 3.6 & 6.1 & 6.7 & 4.5 & 1.5 & 3.6 & 0.6 & 1.0 & 0.7 & 0.6 & 0.1 & 0.1 & 0.3 & 41.4 \\
\hline $\mathrm{NBH} 1 \mathrm{a}$ & 1869 & 0.4 & 1.5 & 1.1 & 0.7 & 1.6 & 0.7 & 0.4 & 0.5 & 0.5 & 0.4 & 0.6 & 0.2 & 0.2 & 0.5 & 1.1 & 0.1 & 0.1 & 1.0 & 11.7 \\
\hline $\mathrm{NBH} 1 \mathrm{a}$ & 1840 & 10.6 & 21.8 & 50.5 & 33.8 & 21.8 & 20.6 & 26.8 & 26.3 & 23.2 & 5.4 & 14.3 & 3.3 & 2.3 & 3.8 & 2.5 & 0.6 & 1.1 & 0.4 & 269.2 \\
\hline $\mathrm{NBH} 1 \mathrm{a}$ & 1836 & 204 & 437 & 1230 & 739 & 604 & 511 & 580 & 587 & 468 & 163 & 307 & 59.4 & 46.6 & 78.7 & 46.3 & 5.84 & 7.34 & 2.21 & 6076 \\
\hline
\end{tabular}


160 Table S3: Results of General Linear Models for dependence of PCB congeners and DDE in tern eggs on year and species (data for

161 1972-2005) and in upper NBH sediment core sample [15]; data from 1971 - 1996 (data for PCB018 and PCB044 are omitted because 162 most of the values for 1994-2005 were below the minimum detection limits (MDLs) for tern eggs, Table S1). Values for Log ${ }_{10} \mathrm{~K}_{\mathrm{ow}}$

163 (the log of the partition coefficient between n-octanol and water) are derived from [16, 17].

\begin{tabular}{|c|c|c|c|c|c|c|c|c|c|c|}
\hline \multirow{2}{*}{ Analyte } & \multicolumn{3}{|c|}{$\mathrm{k}_{1}$} & \multicolumn{2}{|c|}{$\mathrm{k}_{2}$} & \multicolumn{2}{|c|}{$\mathrm{k}_{3}$ (CT effect) } & \multirow{2}{*}{$\begin{array}{c}\mathrm{CT} / \mathrm{RT} \\
\text { ratio }\end{array}$} & \multicolumn{2}{|l|}{$\begin{array}{c}\text { Sediment } \\
\text { Core } \mathrm{k}_{1}\end{array}$} \\
\hline & $\begin{array}{l}\log _{10} \\
\text { Kow}^{*}\end{array}$ & Estimate & p-value & Estimate & p-value & Estimate & p-value & & Estimate & $\begin{array}{c}\mathrm{p}^{-} \\
\text {value }\end{array}$ \\
\hline PCB008 & 5.03 & -0.182 & $<0.0001$ & -0.005 & 0.002 & -0.435 & 0.092 & 0.647 & -0.078 & 0.033 \\
\hline PCB028 & 5.55 & -0.114 & $<0.0001$ & 0.002 & 0.027 & 0.522 & 0.000 & 1.686 & -0.064 & 0.017 \\
\hline PCB052 & 5.86 & -0.193 & $<0.0001$ & -0.003 & 0.089 & 1.486 & $<0.0001$ & 4.422 & -0.048 & 0.040 \\
\hline РCB066 & 6.01 & -0.091 & $<0.0001$ & 0.002 & 0.006 & 0.282 & 0.014 & 1.326 & -0.062 & 0.016 \\
\hline PCB101 & 6.33 & -0.079 & $<0.0001$ & 0.000 & 0.632 & 0.367 & 0.006 & 1.443 & -0.049 & 0.016 \\
\hline PCB105 & 6.39 & -0.076 & $<0.0001$ & 0.002 & 0.004 & 0.198 & 0.038 & 1.219 & -0.036 & 0.047 \\
\hline PCB118 & 6.46 & -0.056 & $<0.0001$ & 0.001 & 0.021 & 0.175 & 0.038 & 1.192 & -0.044 & 0.017 \\
\hline PCB128 & 6.63 & -0.057 & $<0.0001$ & 0.001 & 0.013 & 0.139 & 0.108 & 1.149 & -0.035 & 0.052 \\
\hline PCB138 & 6.71 & -0.043 & $<0.0001$ & 0.001 & 0.000 & 0.107 & 0.116 & 1.113 & -0.035 & 0.030 \\
\hline РCB153 & 6.79 & -0.033 & $<0.0001$ & 0.001 & 0.000 & 0.109 & 0.093 & 1.115 & -0.035 & 0.020 \\
\hline PCB170 & 7.09 & -0.050 & $<0.0001$ & 0.002 & $<0.0001$ & 0.166 & 0.035 & 1.180 & -0.039 & 0.009 \\
\hline PCB180 & 7.17 & -0.040 & $<0.0001$ & 0.002 & $<0.0001$ & 0.165 & 0.015 & 1.179 & -0.038 & 0.011 \\
\hline PCB187 & 7.01 & -0.028 & $<0.0001$ & 0.002 & $<0.0001$ & -0.027 & 0.682 & 0.973 & -0.032 & 0.035 \\
\hline PCB195 & 7.34 & -0.034 & $<0.0001$ & 0.003 & $<0.0001$ & 0.235 & 0.002 & 1.265 & -0.035 & 0.036 \\
\hline PCB206 & 7.8 & -0.005 & 0.430 & 0.004 & $<0.0001$ & 0.129 & 0.078 & 1.137 & -0.033 & 0.010 \\
\hline $\begin{array}{l}\text { PCB209 } \\
\text { Total }\end{array}$ & 7.88 & 0.035 & 0.008 & 0.006 & $<0.0001$ & 0.275 & 0.050 & 1.317 & -0.021 & 0.056 \\
\hline PCBs & & -0.051 & $<0.0001$ & 0.002 & $<0.0001$ & 0.167 & 0.031 & 1.182 & -0.054 & 0.019 \\
\hline$p p^{\prime}-\mathrm{DDE}$ & & -0.052 & $<0.0001$ & 0.001 & 0.004 & 0.378 & $<0.0001$ & 1.459 & & \\
\hline
\end{tabular}


165 Table S4: Principal Components Analysis (PCA) of PCB congener patterns in common (CT) 166 and roseate (RT) tern eggs ( $n=100)$ from Buzzards Bay, MA, USA. The values tabulated are the 167 loadings of each congener on PC1 and PC2, with larger absolute values in bold.

\begin{tabular}{|ccc|}
\hline $\begin{array}{c}\text { PCB } \\
\text { congener }\end{array}$ & PC1 & PC2 \\
\hline \hline PCB008 & 0.066 & -0.011 \\
PCB018 & 0.063 & -0.081 \\
PCB028 & $\mathbf{0 . 4 9 7}$ & -0.225 \\
PCB044 & 0.157 & -0.141 \\
PCB052 & $\mathbf{0 . 4 1 1}$ & -0.032 \\
PCB066 & $\mathbf{0 . 3 5 3}$ & -0.082 \\
PCB101 & $\mathbf{0 . 3 0 1}$ & $\mathbf{0 . 6 7 4}$ \\
PCB105 & $\mathbf{- 0 . 4 5 4}$ & 0.164 \\
PCB118 & -0.003 & $\mathbf{0 . 3 2 1}$ \\
PCB128 & -0.184 & -0.052 \\
PCB138 & -0.220 & 0.107 \\
PCB153 & 0.172 & -0.024 \\
PCB170 & -0.023 & -0.215 \\
PCB180 & -0.114 & $-\mathbf{0 . 4 0 4}$ \\
PCB187 & 0.009 & 0.11 \\
PCB195 & -0.014 & -0.144 \\
PCB206 & -0.053 & -0.196 \\
PCB209 & -0.041 & -0.190 \\
\hline
\end{tabular}

168 
170 Table S5: Numbers of amino acid (above diagonal) and nucleotide (below diagonal) differences 171 in coding sequence of roseate tern (RT) AHR1 variants as compared to the common tern (CT) 172 and chicken $(\mathrm{CH})$ AHR1.

\begin{tabular}{|c|c|c|c|c|c|}
\hline nucleotidelamino acid & RT AHR1*1 & RT AHR1*2 & $\begin{array}{c}\text { RT } \\
\text { AHR1*3 }\end{array}$ & CT AHR1 & $\begin{array}{c}\text { CH } \\
\text { AHR1 }\end{array}$ \\
\hline RT AHR1*1 & & 1 & 6 & 0 & 68 \\
\hline RT AHR1*2 & 1 & & 7 & 1 & 68 \\
\hline RT AHR1*3 & 9 & 10 & & 6 & 74 \\
\hline CT AHR1 & 12 & 13 & 20 & & 68 \\
\hline CH AHR1 & 228 & 225 & 234 & 226 & \\
\hline
\end{tabular}

173

174 
175 Table S6: Numbers of breeding pairs of common (CT) and roseate (RT) terns at the two nesting 176 sites in Buzzards Bay, Massachusetts, that have been included in this study: Bird Island and Ram 177 Island; methods and data sources as described in SI.

\begin{tabular}{|c|c|c|c|c|c|c|c|c|c|}
\hline SPECIES & YEAR & PERIOD & $\begin{array}{l}\text { Bird } \\
\text { Island }\end{array}$ & $\begin{array}{l}\text { Ram } \\
\text { Island }\end{array}$ & SPECIES & YEAR & PERIOD & $\begin{array}{l}\text { Bird } \\
\text { Island }\end{array}$ & $\begin{array}{l}\text { Ram } \\
\text { Island }\end{array}$ \\
\hline $\mathrm{CT}$ & 1952 & 1950 & 3,000 & 2000 & RT & 1952 & 1950 & 1,800 & 1,300 \\
\hline CT & 1953 & 1950 & 3,000 & 2500 & RT & 1953 & 1950 & 1,400 & 1,700 \\
\hline CT & 1954 & 1950 & 2,800 & 1500 & RT & 1954 & 1950 & 1,400 & 1,000 \\
\hline CT & 1955 & 1950 & 2,400 & 2000 & RT & 1955 & 1950 & 1,200 & 1,200 \\
\hline CT & 1956 & 1950 & 2,000 & 2000 & RT & 1956 & 1950 & 1,400 & 1,000 \\
\hline CT & 1968 & 1960 & 200 & 600 & RT & 1968 & 1960 & 400 & 800 \\
\hline CT & 1969 & 1960 & 200 & 500 & RT & 1969 & 1960 & 400 & 700 \\
\hline $\mathrm{CT}$ & 1970 & 1970 & 250 & 300 & RT & 1970 & 1970 & 600 & 700 \\
\hline CT & 1971 & 1970 & 350 & 250 & RT & 1971 & 1970 & 1,300 & 120 \\
\hline $\mathrm{CT}$ & 1972 & 1970 & 310 & 220 & RT & 1972 & 1970 & 1,100 & 170 \\
\hline CT & 1973 & 1970 & 390 & 25 & RT & 1973 & 1970 & 1,600 & 0 \\
\hline CT & 1974 & 1970 & 350 & 72 & RT & 1974 & 1970 & 1,200 & 0 \\
\hline CT & 1975 & 1970 & 400 & 0 & RT & 1975 & 1970 & & \\
\hline CT & 1976 & 1970 & 470 & 0 & RT & 1976 & 1970 & & \\
\hline CT & 1977 & 1970 & 400 & 0 & RT & 1977 & 1970 & 900 & 0 \\
\hline $\mathrm{CT}$ & 1978 & 1970 & 500 & 0 & RT & 1978 & 1970 & 1,290 & 0 \\
\hline $\mathrm{CT}$ & 1979 & 1970 & 550 & 0 & RT & 1979 & 1970 & 1,500 & 0 \\
\hline CT & 1980 & 1980 & 600 & 0 & RT & 1980 & 1980 & 1,400 & 0 \\
\hline $\mathrm{CT}$ & 1981 & 1980 & 600 & 0 & RT & 1981 & 1980 & & \\
\hline CT & 1982 & 1980 & 650 & 0 & RT & 1982 & 1980 & & \\
\hline $\mathrm{CT}$ & 1983 & 1980 & 720 & 0 & RT & 1983 & 1980 & & \\
\hline CT & 1984 & 1980 & 810 & 0 & RT & 1984 & 1980 & 1,650 & 0 \\
\hline $\mathrm{CT}$ & 1985 & 1980 & 1040 & 0 & RT & 1985 & 1980 & 1,450 & 0 \\
\hline CT & 1986 & 1980 & 1129 & 0 & RT & 1986 & 1980 & 1,700 & 0 \\
\hline $\mathrm{CT}$ & 1987 & 1980 & 1337 & 0 & RT & 1987 & 1980 & 1,558 & 0 \\
\hline CT & 1988 & 1980 & 1613 & 0 & RT & 1988 & 1980 & 1,572 & 0 \\
\hline CT & 1989 & 1980 & 1879 & 0 & RT & 1989 & 1980 & 1,473 & 0 \\
\hline CT & 1990 & 1990 & 1803 & 0 & RT & 1990 & 1990 & 1,550 & 0 \\
\hline $\mathrm{CT}$ & 1991 & 1990 & 1780 & 0 & RT & 1991 & 1990 & 1,728 & 0 \\
\hline CT & 1992 & 1990 & 1595 & 2 & RT & 1992 & 1990 & 1,379 & 0 \\
\hline $\mathrm{CT}$ & 1993 & 1990 & 1829 & 98 & RT & 1993 & 1990 & 1,319 & 0 \\
\hline CT & 1994 & 1990 & 1803 & 157 & RT & 1994 & 1990 & 1,314 & 76 \\
\hline $\mathrm{CT}$ & 1995 & 1990 & 1590 & 431 & RT & 1995 & 1990 & 1,447 & 197 \\
\hline $\mathrm{CT}$ & 1996 & 1990 & 1780 & 1085 & RT & 1996 & 1990 & 1,715 & 719 \\
\hline $\mathrm{CT}$ & 1997 & 1990 & 2033 & 920 & RT & 1997 & 1990 & 1,432 & 253 \\
\hline $\mathrm{CT}$ & 1998 & 1990 & 1903 & 1307 & RT & 1998 & 1990 & 1,113 & 543 \\
\hline
\end{tabular}




\begin{tabular}{|l|l|l|l|l|l|l|l|l|l|l|}
\hline CT & 1999 & 1990 & 1836 & 1887 & & RT & 1999 & 1990 & 1,148 & 630 \\
\hline CT & 2000 & 2000 & 1880 & 2030 & & RT & 2000 & 2000 & 1,130 & 988 \\
\hline CT & 2001 & 2000 & 2136 & 2065 & & RT & 2001 & 2000 & 1,062 & 626 \\
\hline CT & 2002 & 2000 & 1702 & 2307 & & RT & 2002 & 2000 & 505 & 952 \\
\hline CT & 2003 & 2000 & 2054 & 1997 & & RT & 2003 & 2000 & 904 & 557 \\
\hline CT & 2004 & 2000 & 1825 & 2938 & & RT & 2004 & 2000 & 554 & 936 \\
\hline CT & 2005 & 2000 & 1857 & 2278 & & RT & 2005 & 2000 & 680 & 724 \\
\hline CT & 2006 & 2000 & 1866 & 2129 & & RT & 2006 & 2000 & 1,111 & 463 \\
\hline CT & 2007 & 2000 & 1863 & 2214 & & RT & 2007 & 2000 & 919 & 661 \\
\hline CT & 2008 & 2000 & 1576 & 2354 & RT & 2008 & 2000 & 747 & 563 \\
\hline
\end{tabular}


181 Figure S1: Representative chromatograms used for quantifying DDE in tern eggs, showing 182 samples collected in 1972 (upper panel) and 1995 (lower panel).
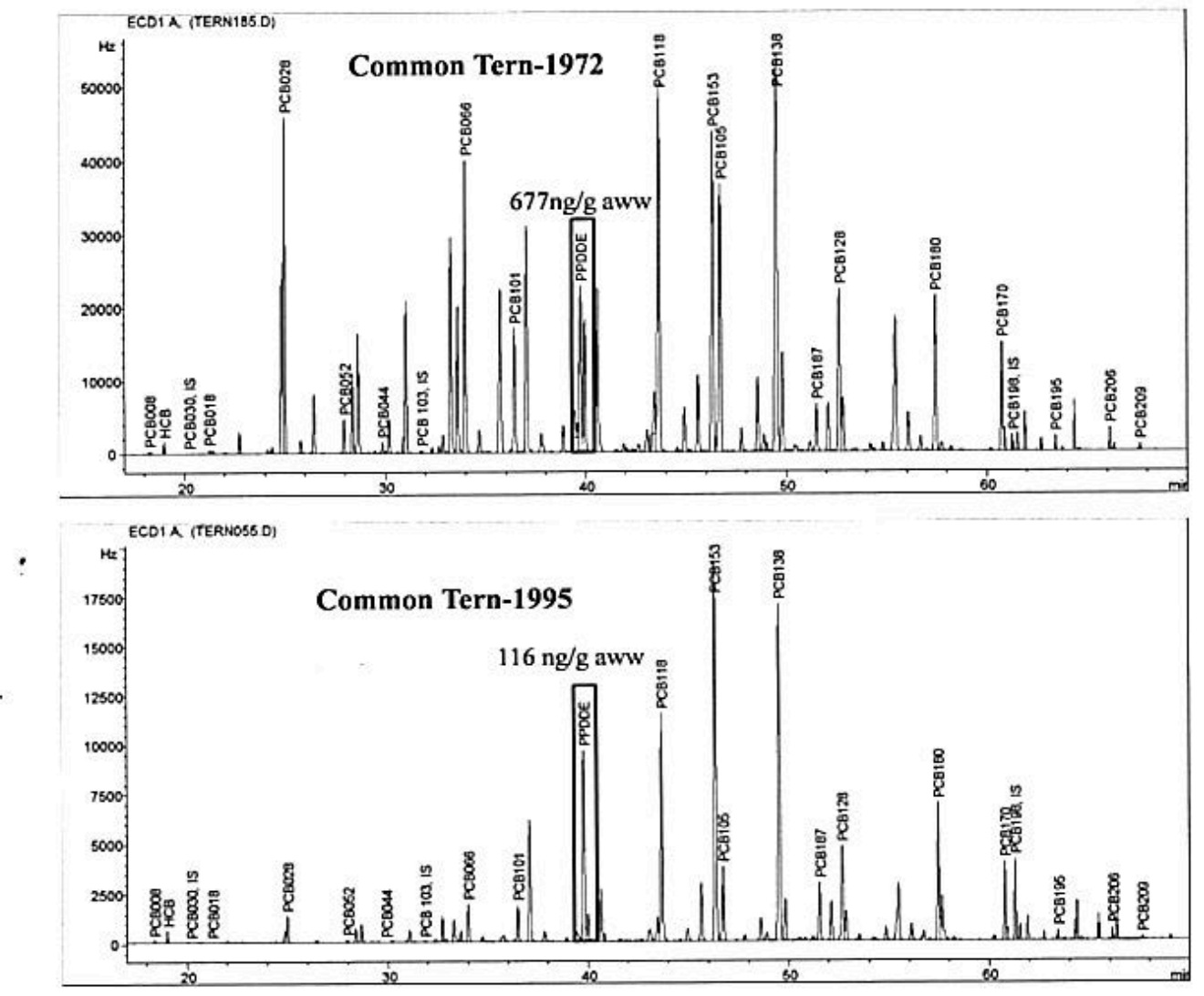

183 
185 Figure S2: Representative samples showing PCB homologue distributions in Aroclors 1016 and 186 1242, New Bedford Harbor (NBH) sediment, and Common Tern (CT) eggs from 1972 and 1995.

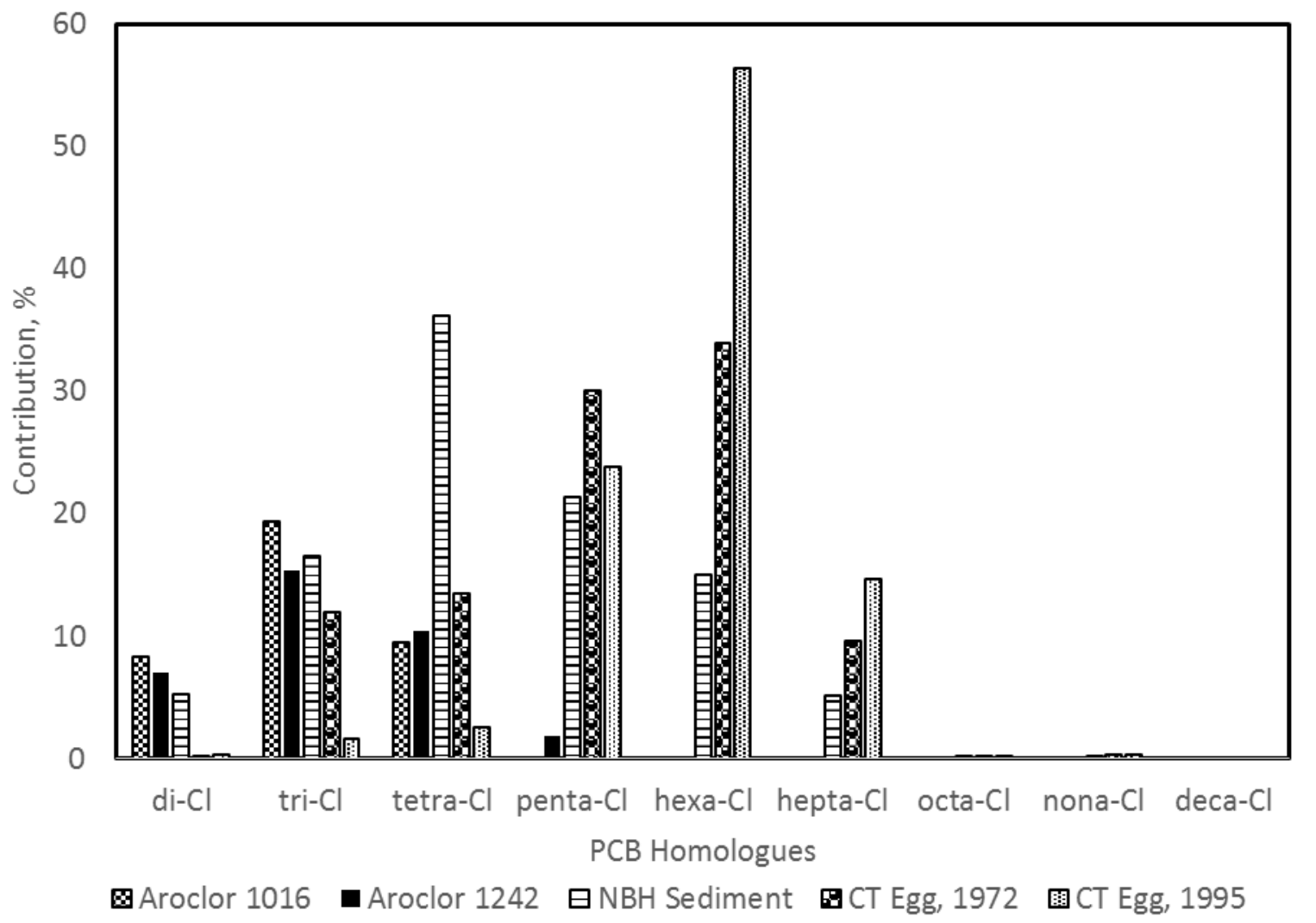


188 Figure S3: Principal Components (PC) plot of PCB congener patterns in single eggs of common 189 terns (CT) and roseate terns (RT) collected from Buzzards Bay in the periods 1970s, 1990s and 190 2000s, where the coordinates of each sample on the PC axes are the principal component scores 191 and the vector length for each congener represents its contribution to the axes (Table S4).

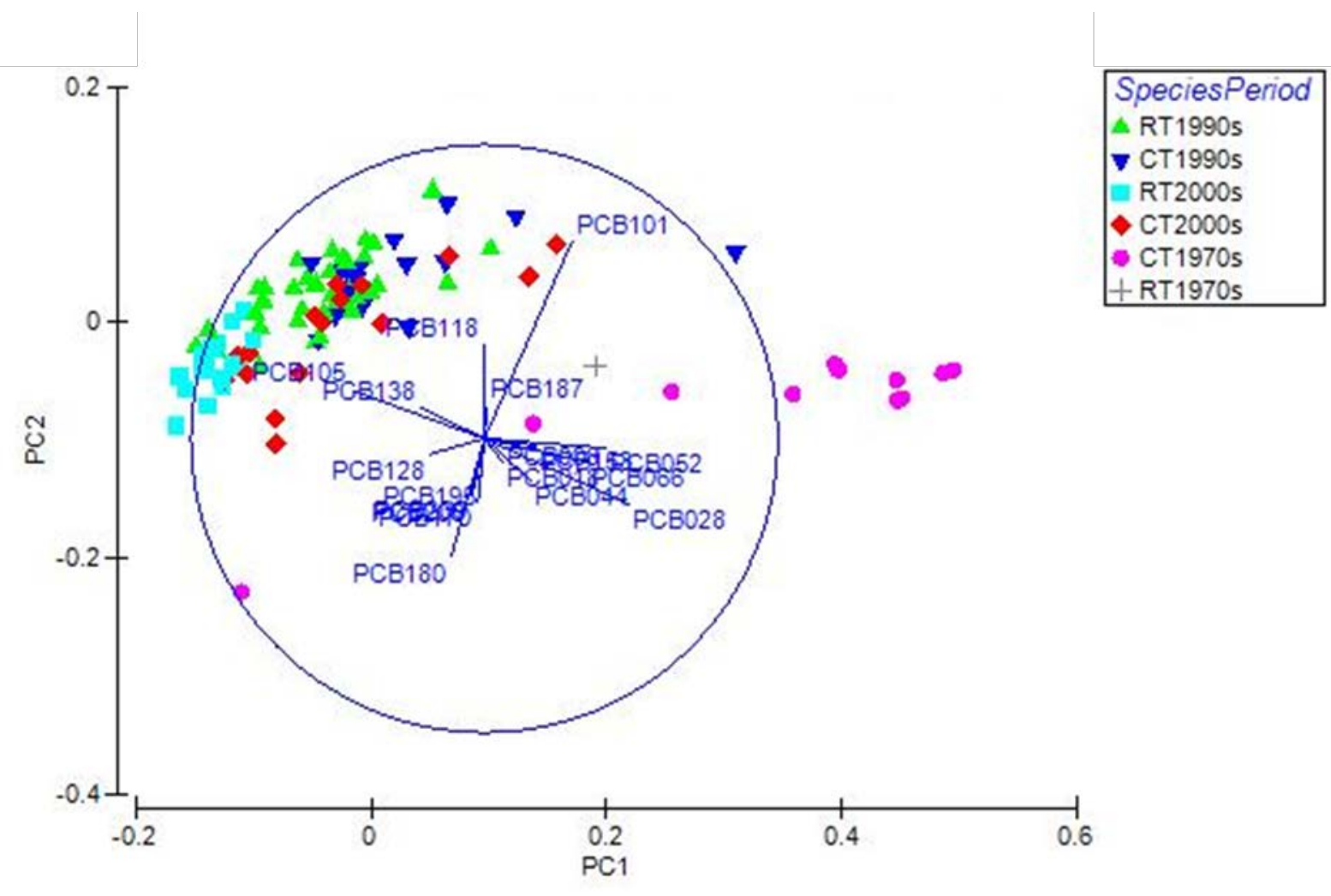

192 
193 Figure S4: Amino acid sequences of roseate tern AHR variants (RT 1*1, RT 1*2, RT 1*3),

194 showing differences from each other and from common terns AHR1 (ShAHR) and from chicken

195 AHR1 (GgAHR).

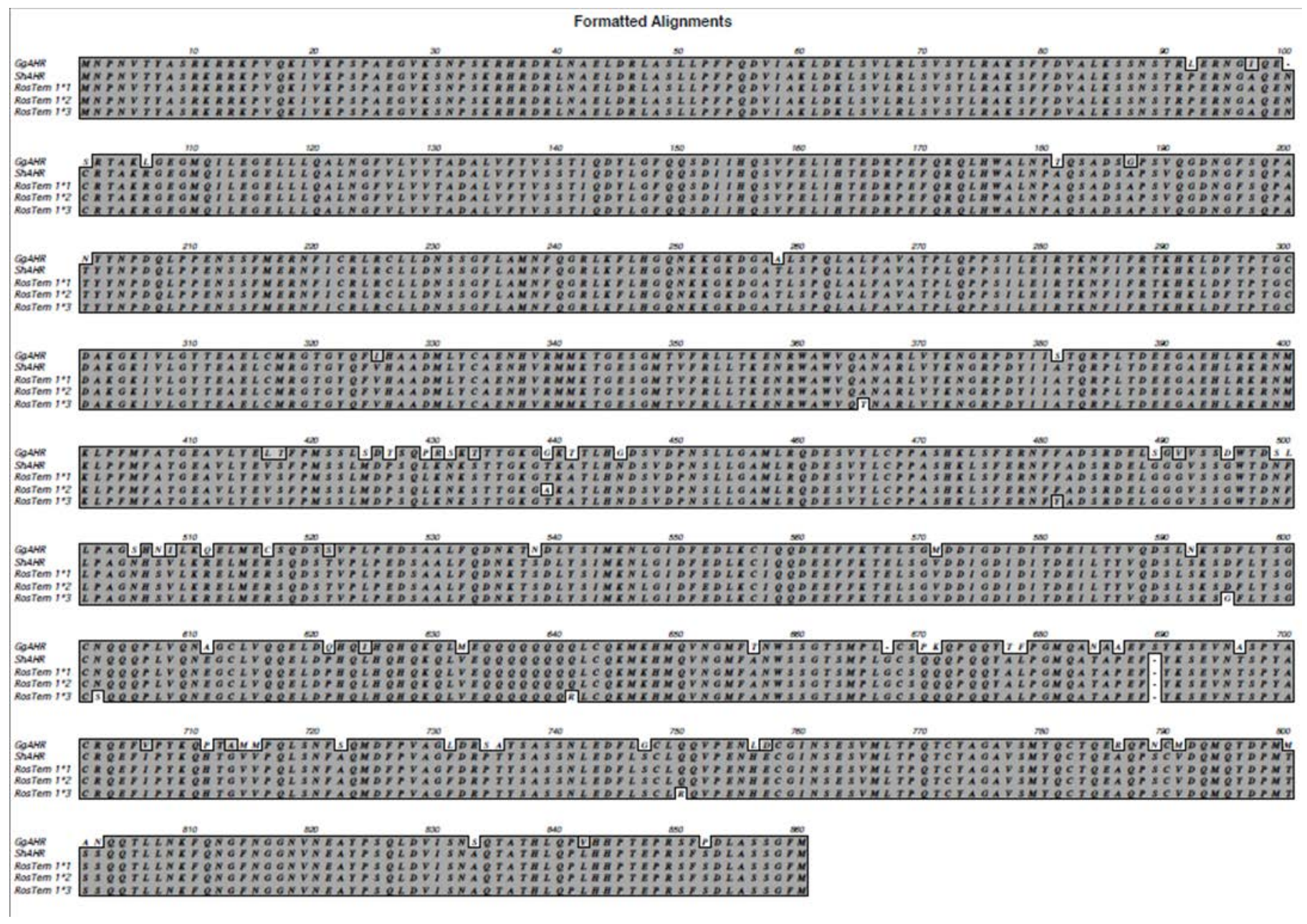

197 
198 Figure S5: Decadal averages ( \pm standard deviations) of the numbers of breeding pairs of 199 common terns (CT, top) and roseate terns (RT, bottom) and concentrations of egg PCBs and 200 DDE ng g-1 adjusted wet weight (aww).
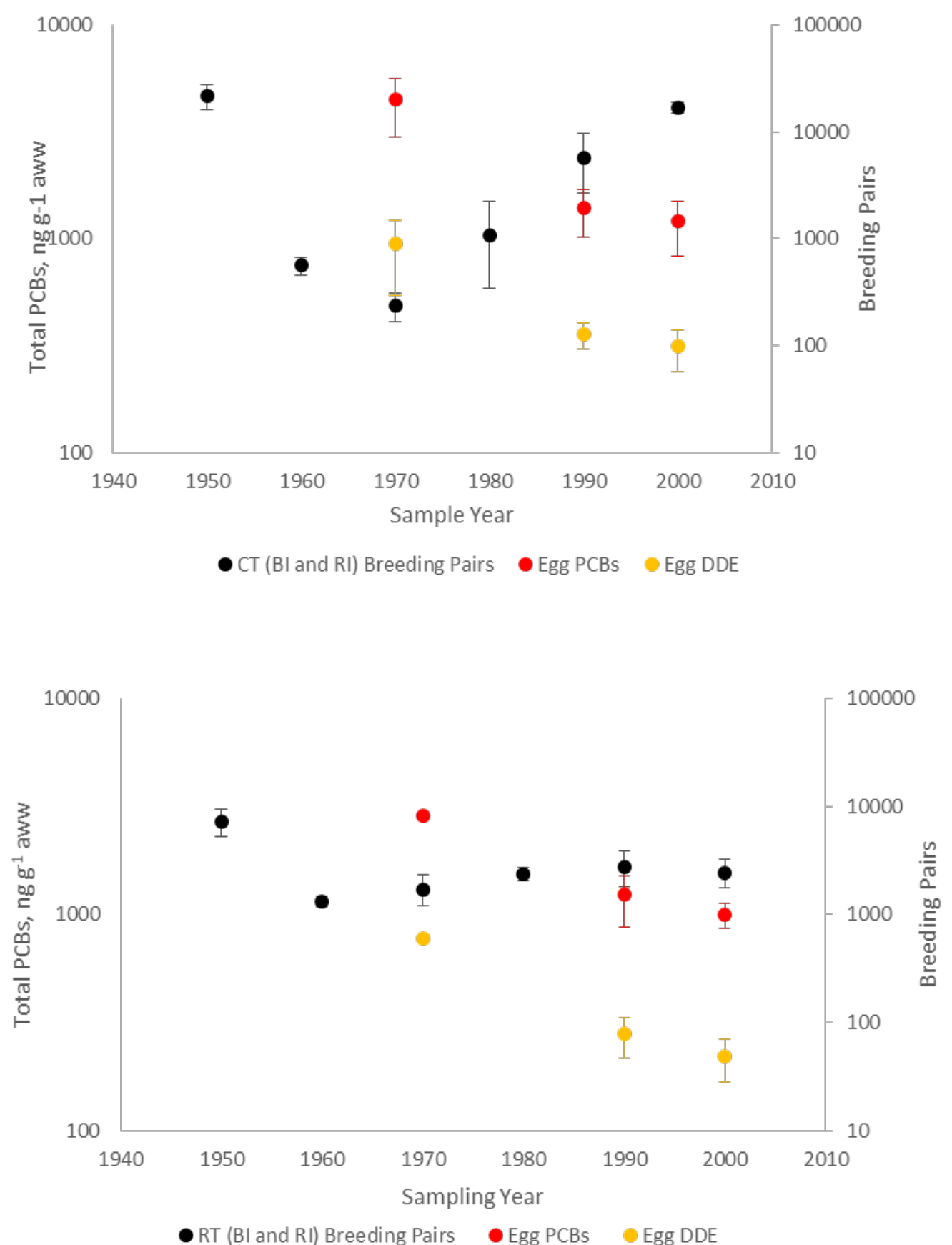